\title{
CDater solakm
}

Technical Report
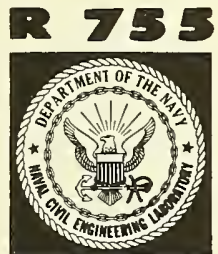

February 1972
NAVAL CIVIL ENGINEERING LABORATORY

Port Hueneme, Califonia 93043

Sponsored by

NAVAL FACILITIES ENGINEERING COMMAND
Robert 6. Walden

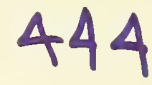

Woods Hote, MA 02543.

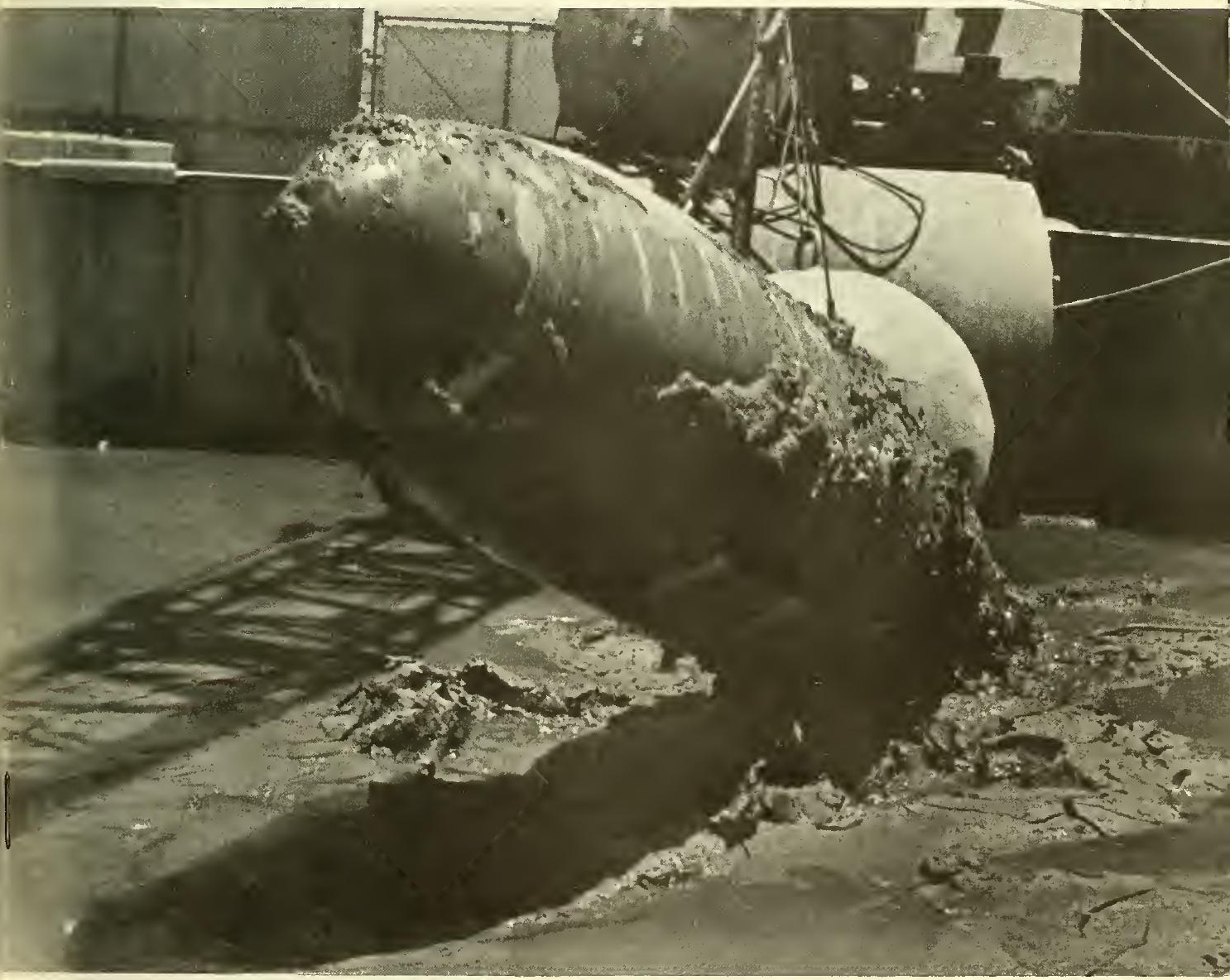

UNAIDED BREAKOUT OF PARTIALLY EMBEDDED OBJECTS FROM COHESIVE SEAFLOOR SOILS $\sqrt{4}$ 


\section{UNAIDED BREAKOUT OF PARTIALLY EMBEDDED OBJECTS FROM COHESIVE SEAFLOOR SOILS}

Technical Report R-755

YF 38.535.002.01.009

by

H. J. Lee

\section{ABSTRACT}

The Naval Civil Engineering Laboratory has conducted field and laboratory tests to investigate the effort required to remove partially embedded objects from cohesive seafloor soils. This work is intended to aid in proper selection of elements for Navy salvage and rescue operations. This report presents the results of the tests and an analysis of the results. Procedures are given for use by field engineers in predicting forces required to remove objects immediately and in estimating times required when lesser forces are applied. The accuracy of the force prediction procedure is about plus or minus 50\%; the accuracy of the time prediction procedure is about plus or minus $100 \%$. These accuracies are comparable to those usually attainable with other time-dependent soil mechanics problems and should be acceptable for typical object retrieval operations. 


\section{CONTENTS}

\section{page}

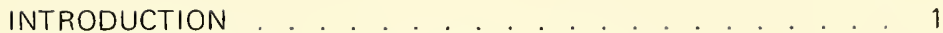

General . . . . . . . . . . . . . . . . 1

Background . . . . . . . . . . . . . . . . . 2

Objectives . . . . . . . . . . . . . . . . 4

TEST PROGRAM AND RESULTS . . . . . . . . . . . . . 4

ANALYSIS . . . . . . . . . . . . . . . . 8

General . . . . . . . . . . . . . . . 8

Immediate Breakout . . . . . . . . . . . . . . 10

Long-Term Breakout . . . . . . . . . . . . . . . . . 19

SUGGESTED PROCEDURE . . . . . . . . . . . . . . 25

SAMPLE PROBLEM . . . . . . . . . . . . . . . . . 30

Problem . . . . . . . . . . . . . 30

Solution . . . . . . . . . . . . . 30

SUMMARY AND CONCLUSIONS . . . . . . . . . . . 32

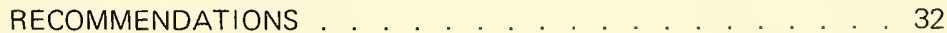

APPENDIXES

A - FY-71 Laboratory Tests . . . . . . . . . . . . . . 34

B - Bearing Capacity Analogy . . . . . . . . . . . . . 38

C - Discussion of Form of Normalized Time Term . . . . . 43

REFERENCES . . . . . . . . . . . . . . . . 46

NOMENCLATURE . . . . . . . . . . . . . . . . . . 49

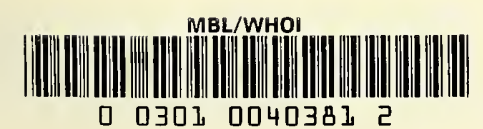





\section{INTRODUCTION}

\section{General}

Objects either partially or completely embedded in soil often require forces greater than their own weight to dislodge them. This additional force requirement, termed the breakout problem, enters into the planning and execution of many Navy undersea operations. Among these are the salvage of sunken vessels and ordnance, the retrieval of bottom-sitting devices or structures, and the use of embedment anchors. In each of these cases, it would be desirable to be able to predict in advance the magnitudes of these breakout forces so that the elements of the operation would be selected and used appropriately.

The overall problem of breakout is very extensive. However, it is possible to divide it into several subproblems which are less broad and, therefore, simpler to study. Division can be made along lines of object embedment depth, soil type, and nature of breakout operation.

A convenient division point for the object embedment depth is the smallest lateral dimension of the object (width). If the object is embedded by an amount greater than its width, breakout usually occurs through failure of the soil above the base of the object. This is the principle of embedment anchor utilization. If the embedment depth is less than the object width, the breakout force problem is mainly concerned with factors below the object base. This latter case will be referred to in this report as the breakout of partially embedded objects.

Soil can be roughly divided into two general types: cohesive and cohesionless. Cohesive soils (clays) are very fine grained and relatively impervious to water flow; cohesionless soils (sands) are coarse grained and very permeable. Shallow breakout from cohesionless soils generally requires only very tittle force in addition to the object weight. The breakout force required with cohesive soils, however, may be substantial.

The nature of the breakout operation can be separated into aided and unaided breakout. Unaided breakout involves solely a direct upward pull on the embedded object; aided breakout includes the performance of other activities in addition to direct pull. Included are water jetting, excavation beneath the object, and rolling of the object prior to force application. 
This report will consider only unaided breakout of partially embedded objects from cohesive seafloor soils. Breakout of embedment anchors ${ }^{1,2}$ and aided breakout of partially embedded objects ${ }^{3,4}$ are considered elsewhere. The results presented in this report are intended to serve as a guide to determine whether aiding is necessary, and, if not, the effort required for unaided breakout.

\section{Background}

Breakout Testing at NCEL. After the loss of the USS Thresher, the Naval Civil Engineering Laboratory (NCEL) initiated a 3-year program to study breakout forces as a part of the Deep Submergence Systems Project (DSSP). The specific objective of this program was to develop procedures for predicting forces required to rescue or salvage sunken vessels such as the Thresher. Unaided breakout of partially embedded objects from cohesive soils was the main area of investigation. During the first year of this program, large-scale field tests were conducted at the Naval Ordnance Test Station, Seal Beach, ocean bottom simulation facility. Various objects, several shaped somewhat like submarines, were placed in a highly heterogeneous clayey silt obtained from nearby borrow areas. The objects were subsequently broken out, and the time and force required were measured. An unsuccessful attempt was made to correlate the experimental results through use of fluid mechanics principles. A major problem involved in making any correlation of these data was found to be the heterogeneity of the soil. It was impossible to assign any consistent series of parameters to the soil being tested. The value of these initial tests was the opportunity they provided to view the breakout mechanism directly. The tests also provided an opportunity to study the effects of lifting an object from one end rather than from its center of mass. This was apparently effective in reducing the required breakout force. ${ }^{5}$

During the second year of the DSSP breakout test program, field tests were conducted in about 30 feet of water in San Francisco Bay near the San Francisco Naval Shipyard at Hunters Point. Objects were partially embedded in the bottom soil and then lifted free. The required breakout forces were provided by a counterweight mounted on a moored barge. Each test consisted of first applying a specified uplift force to a partially embedded object and then measuring the upward displacement of the object as a function of time. Some of the soil properties were obtained from laboratory tests on core samples and also from in-situ shear tests conducted with a diver-held vane device. An empirical equation which related the soil shearing characteristics to the breakout force-time behavior was constructed. ${ }^{6}$ 
Also during the second year of the program, a computer model based on elastoplastic theory was developed for analyzing the breakout problem. The model was formulated in such a way that the force required for essentially instantaneous breakout could be related to the object geometry, embedment depth, and soil shear strength. No time characteristics were considered. ${ }^{2}$

During the third and final year of the DSSP breakout program, field tests were conducted in 100 feet of water off the Louisiana coast in the Gulf of Mexico. The same objects used in the San Francisco Bay tests were partially embedded in the bottom soil and then removed by using inflated pontoons to provide the required forces. The upward displacements of the objects as functions of time after force application were measured in several of the tests. ${ }^{7}$

During the latter portion of the third year of the program, small-scale laboratory tests were conducted at NCEL. These involved placing small objects in a tank containing disturbed cohesive soil obtained from nearby marsh areas. An uplift force was applied, and the total time required for breakout was measured. Intermediate object displacements were not measured. ${ }^{7}$

After the experimental testing, a report describing the tests and listing the test results was prepared. ${ }^{7}$ This report included an empirical equation relating breakout force, breakout time, object geometry, soil strength, and time in place before force application. The correlation was not sufficient for predicting breakout forces with an acceptable degree of accuracy in field operations.

In FY-70 (fiscal year 1970), an analysis of the previous partially embedded object breakout test results was conducted under the sponsorship of the Naval Facilities Engineering Command. This analysis led to the recommendation that additional small-scale laboratory tests were needed to better define the breakout problem. These were conducted in the NCEL Seafloor Soils Laboratory during FY-71 and included approximately 90 tests performed under carefully controlled conditions. The test results have been incorporated into a general framework, discussed in later sections of this report.

Test Work Done Outside NCEL. At least two organizations besides NCEL have conducted unaided breakout tests involving objects partially embedded in cohesive soil. The Southwest Research Institute conducted a series of such tests within a 90-inch-diameter pressure vessel. The results were considered preliminary and were not analyzed in detail. The authors concluded that the breakout force is a function of object size, soil type, and in-situ time and is not a function of the hydrostatic pressure. ${ }^{8}$

The Lockheed Missiles and Space Company performed a large number of breakout tests in a reservoir near Santa Cruz, California, and in a test bin filled with San Francisco Bay mud. The tests were begun by placing such objects as 55-gallon oil drums and scale models of the Navy's DSRV (Deep 
Submergence Rescue Vehicle) into cohesive soil at either the reservoir or test bin sites. The objects were allowed to remain in place for varying times and then broken out either by rapidly increasing the uplift force until the occurrence of breakout or by applying a smalter force and measuring the time required for breakout. The results were presented in the form of regression equations relating breakout force to object embedment depth. These were given for different object shapes and in-situ times. No attempt was made to derive general equations which could be extrapolated to situations other than those investigated. It was concluded, however, that embedment depth is a significant parameter in determining the required breakout force. ${ }^{9}$

\section{Marine Salvage and Offshore Petroleum Industry Operations.}

Documented evidence relative to the problem of freeing objects from the seafloor is limited. A "mud suction" force is often mentioned in the literature of marine salvage operations, but no measurements of such forces seem to have been made. In the offshore petroleum industry, it is often necessary to extract large mat foundations from the seafloor soils. In some cases, the forces required have been measured, but these are difficult to analyze because the mat embedment depth is generally not known and the lifting process is usually accompanied by water jetting beneath the mat. A good review of the breakout problems associated with marine salvage and offshore petroleum industry operations is provided by two NCEL reports. ${ }^{5,6}$

\section{Objectives}

The objectives of this report are:

1. To present the results of NCEL breakout tests.

2. To provide an analysis of these data.

3. To recommend practical techniques for use by field engineers in estimating the effort required to break out partially embedded objects from seafloor soils.

\section{TEST PROGRAM AND RESULTS}

As discussed in the Background section, NCEL has conducted five series of tests to investigate the unaided breakout of partially embedded objects. They are:

1. FY-66 Seal Beach field tests

2. FY-67 San Francisco Bay field tests 
3. FY-68 Gulf of Mexico field tests

4. FY-68 laboratory tests

5. FY-71 laboratory tests

Because the soil at the Seal Beach facility was so heterogeneous, the results were inconsistent and conflicting. The Seal Beach tests, therefore, will not be considered further in this report.

The specific procedural details of the field tests at San Francisco Bay and Gulf of Mexico and the FY-68 laboratory tests are given by two earlier NCEL reports. ${ }^{6,7}$ The procedures followed in the $F Y-71$ laboratory tests are given in Appendix $A$ of this report. The procedures followed in all of these test series can be generalized by these steps:

1. The first step in the performance of a breakout test is the placement of the object into the cohesive soil medium. In the field tests, this was accomplished by merely placing the objects on the soil surface and allowing them to settle under their own weight. In the laboratory tests, the objects were pressed onto the soil to predetermined embedment depths. The objects used in the various tests are listed in Table 1.

2. The second step is a waiting period during which the soil around the object may regain some of the strength lost during penetration and also during which objects may continue to settle. In the San Francisco Bay field tests and FY-68 laboratory tests, the objects were unsupported during this period. In some of the Gulf of Mexico field tests, a constant upward force on a line attached to the object was applied during the waiting period. In all of the FY-71 laboratory tests, an upward line force approximately equal to the object weight was applied so that no further settlement would occur during the waiting period. This was done to maintain the embedment depth as an independent variable. The length of the waiting period will be identified in this report as $t_{w}$ and the total upward line force applied to the objects during this period as $F_{\ell_{\mathbf{w}}}$. (A foldout NOMENCLATURE, after the REFERENCES, gives a complete list of symbols.) The embedment depth at the end of this period will be identified as $D$.

3. The third step is the application of the breakout force. In the NCEL tests, this included as many as three different phases: 
a. During the first phase of all of the field tests and some of the FY.71 laboratory tests, the upward line force on the objects was increased gradually until either breakout occurred or a predetermined value was reached. For the remaining laboratory tests, the first phase consisted of a more or less instantaneous application of the predetermined line force.

b. If breakout did not occur during the first phase, a second phase was begun during which the line force was maintained at a constant value for an indefinite period of time. Breakout usually occurred at some point during this phase, with the length of the time period varying between a few seconds and several weeks.

c. During a few of the field tests, there was also a third phase (after the waiting period of the second phase). which involved a further increase in line force to the point of breakout. This was done whenever it appeared that the test would otherwise extend into the night.

To develop an analysis of these data, it is necessary to reduce the number of pertinent variables to a minimum. This can be done by describing the load application portion of each test by two quantities, a breakout force and a breakout time. In this report, the breakout line force, $F_{\ell b}$, will be taken as that line force applied at the end of the first phase of loading for throughout the second phase for those tests which continue into the second phase). The breakout time, $t_{b}$, will be taken as the length of the second (constant line force) phase of loading plus one-half of the length of the first (variable line force) phase during which the line force exceeds the buoyant weight of the object.

The third phase of loading will be ignored because the results of tests which included a third phase would be too complicated to analyze otherwise. Instead, those tests with a third phase will be considered as tests in which breakout did not occur under a line force, $F_{\ell_{b}}$, applied for a period, $t_{b}$, as defined above. 


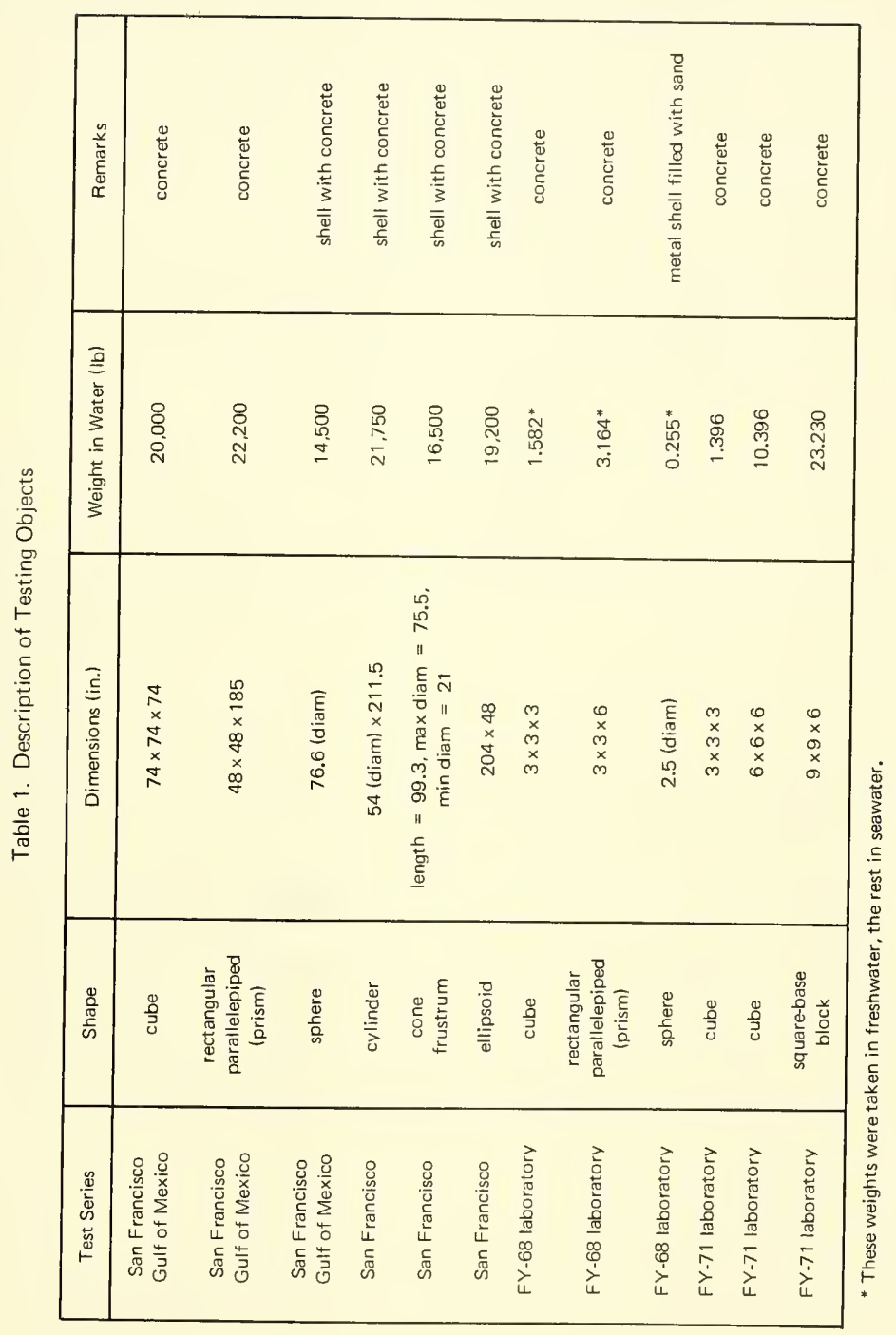


A general compilation of the results from the San Francisco Bay and Gulf of Mexico field tests and the FY-68 and FY-71 laboratory tests is given in Table 2. Some of the terms require further definition as follows:

B - The smallest lateral object dimension (width). For spheres and cylinders, this is taken as the object width at the soil-water interface.

A - Object cross-sectional area. For spheres and cylinders, this is taken at the soil-water interface.

$W_{s}$ - Buoyant weight of soil displaced by object (equal to the buoyant unit weight of soil, $\gamma_{s}$, times the volume of the portion of object embedded in soil, $\mathbf{V}_{\mathbf{s}}$ ).

$W_{b}$ - Buoyant weight of object.

$F_{b}-$ Breakout force carried by soil (equal to $F_{\ell_{b}}-W_{b}+W_{s}$ ).

$\mathrm{F}_{\mathrm{q}}$ - Downward force carried by soil during waiting period prior to breakout loading (equal to $W_{b}-W_{s}-F_{\ell w}$ ).

$\mathrm{D}$ - Embedment depth (assumed equal to $\mathrm{V}_{\mathrm{s}} / \mathrm{A}$ for spheres and cylinders).

$S_{v}$ - Vane shear strength of soil.

The rationale behind the use of $F_{b}$ and $F_{q}$ is discussed in the ANALYSIS section. The value of soil vane shear strength is not given for the field tests because, as will be seen later, the quantity $F_{q}$ is a better measure of the in-situ undrained shear strength. Measurements of vane shear strength at both sites were made, however, and these are listed in References 6 and 7. The vane shear strength for the FY-68 laboratory tests is derived from data presented in Reference 7. The vane shear strength is taken as 3.5 psf for embedment depths, D, less than 2 inches and equal to 5 psf for greater embedment depths. For the FY-71 tests, vane shear strength was measured with a hand vane at eight points in the test tank after each test. The results were averaged according to the procedure outlined in Appendix $A$ to yield one characteristic vane strength for each test.

\section{ANALYSIS}

\section{General}

Breakout is a very complex soil mechanics problem. Two of the features which make it so are: 
1. When a net upward force is applied to an embedded object, and the object does not break out immediately, the force must be balanced by the development of tensile stresses in the underlying soil. Although most soils are incapable of existing in a state of tension indefinitely, most cohesive soils are capable of resisting tension for moderate to long periods of time, depending upon the soil permeability and the overall drainage conditions. Breakout, therefore, is a distinctly time-dependent process; practically any net upward force will dislodge a partially embedded object if enough time is allowed to pass.

2. Breakout is strongly influenced by slight variations and inhomogeneities. For example, a single seam of sand connecting the bottom of a partially embedded object with a source of free water could almost completely eliminate the resistance of the soil to net upward forces on the object. Another object in exactly the same situation without the sand seam might require a very large breakout force or a long breakout time.

Breakout, therefore, is a complex time-dependent problem not unlike the problem of predicting the time-settlement history of foundations. This latter problem has been researched extensively, and it is still difficult to make an accurate prediction. Considering that breakout is even more strongly influenced by slight variations in soil properties and that considerably less research has been directed towards understanding it, it is doubtful that a truly accurate technique for predicting breakout force-breakout time characteristics can be developed. With the amount of test data presently in existence, however, it is possible to derive somewhat approximate techniques for making first-order estimates.

In developing these approximate techniques, it is first necessary to clearly separate the breakout problem into two subproblems: immediate breakout and long-term breakout.

Immediate breakout is defined in soil mechanics terms as breakout under short-term, undrained loading conditions. Loading is so rapid that no significant amount of water is allowed to flow into or through the underlying soil. In practical terms, immediate breakout occurs when the upward load on the embedded object is increased as rapidly as the available equipment will permit until the object breaks free of the seafloor.

Long-term breakout is defined as breakout occurring under the influence of a force less than the immediate breakout force. A certain amount of time must pass before this smaller force will accomplish what the larger force accomplished in very little time. Long-term breakout is of interest because immediate breakout often requires a large amount of equipment to achieve. It may often be more economical to apply a small force and wait for a period of time (perhaps several hours or even a few days). In soil mechanics terms, long-term breakout corresponds to partially drained or shear creep loading. 
4 It is important to note that for immediate breakout the quantity which must be predicted (the dependent variable) is the immediate breakout force, whereas for long-term breakout the dependent variable is the breakout time.

The analysis of the breakout problem will begin with the subproblem of predicting the immediate breakout force.

\section{Immediate Breakout}

The immediate breakout of partially embedded objects from cohesive soils has many elements in common with the short-term bearing capacity of small footings. In the case of breakout, if water is not permitted to flow into the space created below the object as it moves upward (as it will not if the terms of the definition of immediate breakout apply), then the soil itself must flow into the space. The shearing pattern of the soil flowing into the space will be similar to the shearing pattern of soil flowing away from an object penetrating the ground during a bearing capacity failure. A discussion of the technical aspects of this analogy is presented in Appendix B. In this appendix, it is concluded that a rough first approximation of the immediate breakout force may be obtained with a bearing capacity equation of the form

$$
\frac{F_{I b}}{A}=5 S\left(1.0+0.2 \frac{D}{B}\right)\left(1.0+0.2 \frac{B}{L}\right)
$$

where $\quad F_{I b}=$ portion of immediate breakout line force carried by soilhereafter referred to as the immediate breakout force

$$
=F_{\ell I b}-W_{b}+W_{s}
$$

- $F_{\ell \mathrm{Ib}}=$ immediate breakout line force

$\mathrm{S}=$ representative undrained shear strength of soil (assumed equal to $\mathrm{S}_{\mathrm{v}}$, the vane shear strength)

$\mathrm{L} \quad=$ object length

Other terms are as defined previously. (See page 8.)

For practically all of the NCEL tests, the objects were so shaped that $B$ was equal to $L$.

For those objects, Equation 1 reduces to

$$
\frac{F_{I b}}{A S}=6\left(1.0+0.2 \frac{D}{B}\right)
$$


Table 2 Test Results

\begin{tabular}{|c|c|c|c|c|c|c|c|c|c|c|c|c|c|c|}
\hline Test Sertes & Test No. & Obyect Shape & $\begin{array}{l}\text { Waiting Time, } \\
\text { 'w }(\text { min) }\end{array}$ & $\begin{array}{l}\text { Wanting Time } \\
\text { Line Force, } \\
F_{\left(E_{w}\right.}(1 \mathrm{~b})\end{array}$ & $\begin{array}{c}\text { Embecdment Depth. } \\
\text { D (it) }\end{array}$ & $\begin{array}{l}\text { Obiect Width. } \\
\qquad 8(\pi)\end{array}$ & $\begin{array}{l}\text { Cross:Sectional } \\
\text { Area. A }\left(H_{1} 2\right)\end{array}$ & $\begin{array}{l}\text { Displaced Solt } \\
\text { 8uovant Weight } \\
W_{5}(10)\end{array}$ & $\begin{array}{l}\text { Obyect Werght } \\
\text { in water. } \\
w_{b}(10)\end{array}$ & $\begin{array}{l}\text { Breakour Line } \\
\left.\text { Force, } F_{(b}|l| b\right)\end{array}$ & $\begin{array}{l}8 \text { reakout Fordt } \\
\left(F_{R_{b}}-W_{b}+W_{s}\right) \\
F_{b}(1 b)\end{array}$ & $\begin{array}{l}\text { Sal Vane } \\
\text { Shesr Strengith } \\
\mathrm{S}_{\mathrm{v}}\left(1 \mathrm{~b} / \mathrm{t}^{2} \mathrm{t}^{2}\right)\end{array}$ & 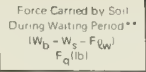 & 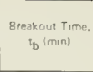 \\
\hline San francisco & $\begin{array}{l}28 \\
24 \\
54 \\
51 \\
38 \\
42 \\
43 \\
50 \\
36 \\
37 \\
46 \\
53 \\
14 \\
27 \\
52 \\
33 \\
\end{array}$ & $\begin{array}{l}\text { soliere } \\
\text { cylinder } \\
\text { cylinder } \\
\text { prisis } \\
\text { cube } \\
\text { cube } \\
\text { woee } \\
\text { cube } \\
\text { prism } \\
\text { prism } \\
\text { orism } \\
\text { pissm } \\
\text { sphere } \\
\text { sphere } \\
\text { cylander } \\
\text { cylinder }\end{array}$ & $\begin{array}{r}720 \\
6.120 \\
2.520 \\
2.520 \\
4.080 \\
5.580 \\
5.760 \\
2.640 \\
1.080 \\
960 \\
14.400 \\
2.580 \\
1020 \\
2.160 \\
1.440 \\
1.260 \\
\end{array}$ & $\begin{array}{l}- \\
- \\
- \\
- \\
- \\
- \\
- \\
- \\
- \\
- \\
- \\
- \\
-\end{array}$ & $\begin{array}{l}1.27 \\
176 \\
2.09 \\
2.25 \\
3.00 \\
4.50 \\
4.83 \\
3.50 \\
1.50 \\
1.75 \\
2.00 \\
2.83 \\
194 \\
178 \\
1.85 \\
209 \\
\end{array}$ & $\begin{array}{l}6.05 \\
450 \\
450 \\
4.00 \\
6.16 \\
6.16 \\
6.16 \\
6.16 \\
400 \\
4.00 \\
4.00 \\
400 \\
6.36 \\
6.34 \\
4.51 \\
451 \\
\end{array}$ & $\begin{array}{l}2872 \\
79.31 \\
7931 \\
61.60 \\
37.95 \\
37.95 \\
37.95 \\
37.95 \\
61.00 \\
6150 \\
6160 \\
61.50 \\
3190 \\
31.50 \\
7930 \\
7930\end{array}$ & $\begin{array}{l}1.092 \\
4.200 \\
4.980 \\
4,380 \\
3.480 \\
5.123 \\
5.498 \\
5.984 \\
2.772 \\
3234 \\
3.696 \\
5.229 \\
1.857 \\
1,698 \\
4.380 \\
4.980 \\
\end{array}$ & $\begin{array}{l}14,600 \\
21,200 \\
21.200 \\
22.200 \\
20,000 \\
20000 \\
20000 \\
20000 \\
22,200 \\
22200 \\
22.200 \\
22,200 \\
14,600 \\
14,600 \\
21.200 \\
21.200\end{array}$ & $\begin{array}{l}18.000 \\
24.200 \\
26.000 \\
24.600 \\
30.300 \\
27,200 \\
25,300 \\
22,500 \\
26,500 \\
27,600 \\
26,300 \\
23,900 \\
16000 \\
19.600 \\
25.000 \\
21,700 \\
\end{array}$ & $\begin{array}{r}4.492 \\
7.200 \\
9.780 \\
6.780 \\
13.780 \\
2.323 \\
10.798 \\
6.484 \\
7.072 \\
8.634 \\
7.796 \\
6.929 \\
3257 \\
6,698 \\
8.180 \\
5.480 \\
\end{array}$ & $\begin{array}{l}- \\
- \\
- \\
- \\
- \\
- \\
- \\
-\end{array}$ & $\begin{array}{l}13.508 \\
17.000 \\
162220 \\
17.820 \\
16.520 \\
14.877 \\
145512 \\
16.016 \\
19.428 \\
18.966 \\
18.504 \\
16.971 \\
12743 \\
12.902 \\
16.820 \\
16220\end{array}$ & $\begin{array}{r}11 \\
6 \\
7 \\
13 \\
21 \\
129 \\
71 \\
715 \\
365 \\
130 \\
899 \\
-\quad 190 \\
7250 \\
285 \\
55 \\
135 \\
175\end{array}$ \\
\hline Sulf of Mexict & $\begin{array}{r}3 \\
5 \\
6 \\
8 \\
9 \\
9 \\
10 \\
14 \\
16 \\
17 \\
22 \\
28 \\
4 \\
13 \\
15 \\
18 \\
19 \\
20 \\
23 \\
25 \\
26 \\
27 \\
\end{array}$ & $\begin{array}{l}\text { iube } \\
\text { cube } \\
\text { cube } \\
\text { spherert } \\
\text { sphere } \\
\text { sphere } \\
\text { sphere } \\
\text { sphere } \\
\text { sphere } \\
\text { spiere } \\
\text { prism } \\
\text { cube } \\
\text { sphere } \\
\text { sphere } \\
\text { sphere } \\
\text { sphere } \\
\text { sphere } \\
\text { sphere } \\
\text { pistsm } \\
\text { prism } \\
\text { cube }\end{array}$ & $\begin{array}{r}1.284 \\
843 \\
422 \\
693 \\
1.357 \\
850 \\
1308 \\
3.750 \\
240 \\
1.011 \\
2.812 \\
2.965 \\
4,122 \\
126 \\
2.481 \\
126 \\
729 \\
600 \\
36,000 \\
832 \\
2.867\end{array}$ & $\begin{array}{c}- \\
10,000 \\
2.000 \\
- \\
= \\
- \\
4,500 \\
5,000 \\
700 \\
600 \\
500 \\
= \\
= \\
- \\
= \\
= \\
= \\
= \\
= \\
-\end{array}$ & $\begin{array}{l}183 \\
033 \\
066 \\
169 \\
1.52 \\
133 \\
133 \\
139 \\
115 \\
0.88 \\
088 \\
075 \\
053 \\
159 \\
147 \\
121 \\
1.86 \\
103 \\
062 \\
033 \\
100\end{array}$ & $\begin{array}{l}616 \\
616 \\
616 \\
630 \\
623 \\
610 \\
610 \\
615 \\
5192 \\
551 \\
400 \\
616 \\
463 \\
602 \\
5097 \\
5.76 \\
6.18 \\
5.74 \\
400 \\
400 \\
616 \\
\end{array}$ & $\begin{array}{l}37.95 \\
3795 \\
37.95 \\
31.27 \\
3052 \\
2922 \\
2922 \\
2969 \\
2754 \\
2388 \\
6160 \\
37.94 \\
1683 \\
28.50 \\
2797 \\
2604 \\
29.95 \\
25.87 \\
6160 \\
6160 \\
3794 \\
194\end{array}$ & $\begin{array}{r}2.638 \\
475 \\
951 \\
2.002 \\
1.759 \\
1.474 \\
1.474 \\
1.569 \\
1.205 \\
797 \\
2.048 \\
1.081 \\
339 \\
1.731 \\
1.559 \\
628 \\
2.127 \\
1.008 \\
1.451 \\
772 \\
1.442 \\
\end{array}$ & $\begin{array}{l}20.001 \\
20000 \\
20,000 \\
14.500 \\
14,500 \\
14,500 \\
14,500 \\
14,500 \\
14.500 \\
14,500 \\
22.200 \\
20.000 \\
14.500 \\
14,500 \\
14.500 \\
14.500 \\
14.500 \\
14.500 \\
22,200 \\
22,200 \\
20.000\end{array}$ & $\begin{array}{l}36.000 \\
21.500 \\
26.000 \\
18.000 \\
22.000 \\
22.000 \\
17.500 \\
21.000 \\
16.600 \\
20.800 \\
26.000 \\
26.000 \\
17.000 \\
16.500 \\
21.300 \\
15.000 \\
18.837 \\
15.000 \\
24.000 \\
23.200 \\
22.000 \\
\end{array}$ & $\begin{array}{l}18.638 \\
1.979 \\
6.951 \\
5.502 \\
9.259 \\
8.974 \\
4.474 \\
8.069 \\
3.305 \\
7.097 \\
5.848 \\
7.081 \\
2.839 \\
3.731 \\
8.359 \\
1.128 \\
6.464 \\
1.508 \\
3.251 \\
1.772 \\
3.442 \\
\end{array}$ & $\begin{array}{l}- \\
\vdots \\
-\end{array}$ & $\begin{array}{r}17.362 \\
9.525 \\
17.049 \\
12.498 \\
12.741 \\
13.026 \\
8.526 \\
7.931 \\
12.595 \\
13.103 \\
19.652 \\
18.919 \\
14.161 \\
12.769 \\
12.941 \\
13.872 \\
11.373 \\
13.992 \\
20.749 \\
21.428 \\
18.558\end{array}$ & $\begin{array}{c}2 \\
05 \\
17 \\
15 \\
4 \\
16 \\
4 \\
2 \\
3 \\
3 \\
15 \\
3 \\
28 \\
142 \\
148 \\
11 \\
542 \\
13 \\
586 \\
304 \\
94 \\
124\end{array}$ \\
\hline FV 68 latboratory & $\begin{array}{r}151 \\
30 \\
37 \\
38 \\
39 \\
40\end{array}$ & $\begin{array}{l}\text { cube } \\
\text { cube } \\
\text { cube } \\
\text { cube } \\
\text { cube } \\
\text { cube }\end{array}$ & $\begin{array}{r}1355 \\
22 \\
17 \\
17 \\
15 \\
16\end{array}$ & $\begin{array}{l}- \\
\overline{-} \\
\overline{-}\end{array}$ & $\begin{array}{l}0.26 \\
0.10 \\
0.10 \\
0.08 \\
0.11 \\
0.13\end{array}$ & $\begin{array}{l}0.25 \\
0.25 \\
025 \\
0.25 \\
0.25 \\
0.25\end{array}$ & $\begin{array}{l}0.0625 \\
00625 \\
0.0625 \\
00625 \\
00025 \\
0.0625\end{array}$ & $\begin{array}{l}0.491 \\
0188 \\
0188 \\
0.156 \\
0.202 \\
0.234\end{array}$ & $\begin{array}{l}1582 \\
1582 \\
1582 \\
1582 \\
1582 \\
1582\end{array}$ & $\begin{array}{l}234 \\
310 \\
280 \\
2.40 \\
200 \\
200\end{array}$ & $\begin{array}{l}125 \\
171 \\
141 \\
0.97 \\
062 \\
065\end{array}$ & $\begin{array}{l}50 \\
3.5 \\
3.5 \\
35 \\
35 \\
35\end{array}$ & $\begin{array}{l}\overline{-} \\
\overline{-} \\
z \\
-\end{array}$ & $\begin{array}{l}00 \\
0.1 \\
01 \\
05 \\
47 \\
67\end{array}$ \\
\hline
\end{tabular}


Table 2. Continued

\begin{tabular}{|c|c|c|c|c|c|c|c|c|c|c|c|c|c|c|}
\hline Test Sertes & Tes! No* & Oofect Shape & $\begin{array}{l}\text { Wartung Time } \\
\text { 'w irmint }\end{array}$ & $\begin{array}{c}\text { Walung Turne } \\
\text { Line Force. } \\
F_{\mathrm{lw}_{w}}(1 \mathrm{~b})\end{array}$ & $\begin{array}{l}\text { Embedment Depth } \\
\quad 0 \text { |ft) }\end{array}$ & $\begin{array}{l}\text { Object Width. } \\
\text { B }\{\text { tht }\end{array}$ & $\begin{array}{l}\text { Cross Sectional } \\
\text { Area. A }\left(\mathrm{ft}^{2}\right)\end{array}$ & $\begin{array}{c}\text { Otsplaced Soil } \\
\text { Buoyant Weight, } \\
W_{g} \text { (it) }\end{array}$ & $\begin{array}{l}\text { Object Werght } \\
\text { in water. } \\
w_{b}(16)\end{array}$ & $\begin{array}{l}\text { Breal out Line } \\
\text { Force. F Fo } 1 \mid \text { | }\end{array}$ & 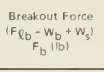 & $\begin{array}{l}\text { Soil Vane } \\
\text { Shear Strength } \\
\mathrm{S}_{\mathrm{y}}\left|1 \mathrm{~b} / \mathrm{t}^{2}\right|\end{array}$ & $\begin{array}{c}\text { Force Carried by Soll } \\
\text { During Warting Period.". } \\
\left(W_{b}-W_{s}-F_{(w)}\right. \\
F_{q}(11 b)\end{array}$ & $\begin{array}{l}\text { Breat oul Time. } \\
\text { tb }\end{array}$ \\
\hline $\begin{array}{l}\text { FY.68 taboratory } \\
\text { (continued) }\end{array}$ & $\begin{array}{r}41 \\
44 \\
43 \\
1 \\
5 \\
7 \\
22 \\
\end{array}$ & $\begin{array}{l}\text { cube } \\
\text { cube } \\
\text { cube } \\
\text { cube } \\
\text { pissm } \\
\text { cube } \\
\text { cube }\end{array}$ & $\begin{array}{r}175 \\
15 \\
20 \\
830 \\
11.480 \\
232 \\
1.027\end{array}$ & $\begin{array}{l}- \\
- \\
y \\
- \\
-\end{array}$ & $\begin{array}{l}0.09 \\
011 \\
0.08 \\
0.18 \\
0.18 \\
0.25 \\
0.25\end{array}$ & $\begin{array}{l}0.25 \\
0.25 \\
0.25 \\
025 \\
025 \\
025 \\
0.25\end{array}$ & $\begin{array}{l}00625 \\
0.0625 \\
0.0625 \\
0.0625 \\
01250 \\
0.0625 \\
0.0625\end{array}$ & $\begin{array}{l}0173 \\
0195 \\
0156 \\
0343 \\
0686 \\
0469 \\
0469\end{array}$ & $\begin{array}{l}1582 \\
1582 \\
1582 \\
1582 \\
3164 \\
1582 \\
1582 \\
\end{array}$ & $\begin{array}{l}2.20 \\
180 \\
1.80 \\
100 \\
3.80 \\
248 \\
218 \\
\end{array}$ & $\begin{array}{l}0.80 \\
0.41 \\
0.37 \\
0.66 \\
132 \\
1.37 \\
1.07\end{array}$ & $\begin{array}{l}3.5 \\
35 \\
3.5 \\
5.0 \\
5.0 \\
5.0 \\
50 \\
\end{array}$ & $\begin{array}{l}- \\
- \\
- \\
- \\
z\end{array}$ & $\begin{array}{r}0.4 \\
22 \\
7 \\
227 \\
-11.460 \\
232 \\
38\end{array}$ \\
\hline FY 71 labaratory & $\begin{array}{l}1.1 \\
1.2 \\
1.3 \\
1.4 \\
1.5 \\
1.6 \\
1.7 \\
1.8 \\
1.9 \\
1.9 \\
110 \\
111 \\
112 \\
113 \\
1.14 \\
1.15 \\
1.16 \\
1.17 \\
1.18 \\
1.19 \\
1.20 \\
1.21 \\
1.22 \\
1.23 \\
1.24 \\
1.25 \\
1.26 \\
1.27 \\
1.28 \\
1.29 \\
1.30 \\
1.32 \\
1.33 \\
1.34 \\
1.35 \\
1.36 \\
1.37 \\
1.38 \\
1.39 \\
1.40\end{array}$ & $\begin{array}{l}\text { cube } \\
\text { cube } \\
\text { cube } \\
\text { cube } \\
\text { cube } \\
\text { cube } \\
\text { cube } \\
\text { cube } \\
\text { cube } \\
\text { cube } \\
\text { cube } \\
\text { cube } \\
\text { cube } \\
\text { cube } \\
\text { cube } \\
\text { cube } \\
\text { cube } \\
\text { cube } \\
\text { cube } \\
\text { cube } \\
\text { cube } \\
\text { cube } \\
\text { cube } \\
\text { cube } \\
\text { cube } \\
\text { cube } \\
\text { cube } \\
\text { cubse } \\
\text { cube } \\
\text { cube } \\
\text { cube } \\
\text { cube } \\
\text { cube } \\
\text { cube } \\
\text { cube } \\
\text { cube } \\
\text { cube } \\
\text { cube } \\
\text { cube }\end{array}$ & 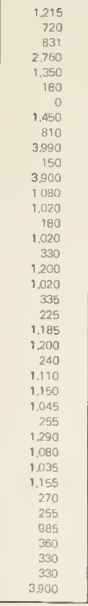 & 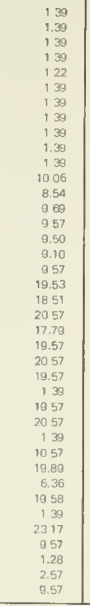 & $\begin{array}{l}0.10 \\
0.08 \\
010 \\
0.08 \\
0.10 \\
0113 \\
0.25 \\
0.25 \\
0.25 \\
0.13 \\
013 \\
0.17 \\
0.25 \\
012 \\
0.08 \\
0.25 \\
0.10 \\
0.25 \\
0.25 \\
008 \\
038 \\
0.08 \\
0.25 \\
0.38 \\
0.38 \\
0.25 \\
0.08 \\
0.08 \\
0.02 \\
0.02 \\
0.02 \\
0.02 \\
0.02 \\
0.02 \\
0.00 \\
0.00 \\
000 \\
039 \\
0.06\end{array}$ & $\begin{array}{l}0.25 \\
0.25 \\
0.25 \\
0.25 \\
0.25 \\
0.25 \\
0.25 \\
0.25 \\
0.25 \\
0.25 \\
0.25 \\
050 \\
0.50 \\
0.50 \\
050 \\
0.50 \\
0.50 \\
0.50 \\
075 \\
075 \\
0.75 \\
0.75 \\
075 \\
0.75 \\
075 \\
0.25 \\
0.75 \\
075 \\
0.25 \\
0.50 \\
075 \\
0.50 \\
075 \\
0.25 \\
0.75 \\
0.50 \\
0.25 \\
050 \\
0.50\end{array}$ & $\begin{array}{l}0.0625 \\
0.0625 \\
0.06655 \\
00025 \\
00625 \\
0.0625 \\
00025 \\
0.0625 \\
0.0625 \\
0.0025 \\
00625 \\
0.2500 \\
0.2500 \\
0.2500 \\
0.2500 \\
0.2500 \\
0.2500 \\
0.2500 \\
0.5625 \\
0.5625 \\
0.5625 \\
0.5625 \\
0.5625 \\
0.5625 \\
0.5625 \\
0.0625 \\
0.5625 \\
0.5625 \\
0.0025 \\
0.2500 \\
0.5625 \\
0.2500 \\
0.5625 \\
0.0625 \\
0.5625 \\
0.2500 \\
00625 \\
0.2500 \\
0.2500\end{array}$ & $\begin{array}{l}0.26 \\
0.21 \\
0.26 \\
0.21 \\
0.26 \\
0.31 \\
0.63 \\
0.63 \\
0.63 \\
0.31 \\
0.31 \\
1.67 \\
250 \\
117 \\
0.83 \\
2.50 \\
0.96 \\
2.50 \\
562 \\
1.87 \\
8.44 \\
1.87 \\
5.62 \\
8.88 \\
8.44 \\
0.03 \\
1.87 \\
1.87 \\
0.05 \\
0.21 \\
0.47 \\
0.21 \\
0.47 \\
0.05 \\
0.00 \\
0.00 \\
0.00 \\
3.75 \\
0.00\end{array}$ & $\begin{array}{r}1396 \\
1.396 \\
1396 \\
1396 \\
1396 \\
1396 \\
1396 \\
1396 \\
1396 \\
1396 \\
1396 \\
10396 \\
103966 \\
10396 \\
10.396 \\
10.396 \\
10.396 \\
10396 \\
23230 \\
23230 \\
23230 \\
23.230 \\
23.230 \\
23230 \\
23230 \\
1.396 \\
23.230 \\
23.230 \\
1396 \\
10396 \\
23230 \\
10.396 \\
23230 \\
1.396 \\
23230 \\
10396 \\
1396 \\
10.396 \\
10396\end{array}$ & $\begin{array}{r}4.14 \\
393 \\
529 \\
701 \\
5.53 \\
536 \\
620 \\
887 \\
80.65 \\
614 \\
496 \\
2241 \\
25.29 \\
2164 \\
17.28 \\
3448 \\
2277 \\
32.83 \\
4308 \\
4212 \\
5636 \\
6991 \\
7389 \\
7932 \\
5743 \\
628 \\
3792 \\
35.63 \\
2.15 \\
1276 \\
2663 \\
13.16 \\
2673 \\
202 \\
24.75 \\
11.18 \\
1.56 \\
2735 \\
11.82\end{array}$ & $\begin{array}{r}3.00 \\
2.74 \\
416 \\
582 \\
476 \\
428 \\
5.42 \\
710 \\
987 \\
406 \\
387 \\
13.57 \\
1739 \\
1241 \\
7.71 \\
27.58 \\
13.33 \\
24.93 \\
25.48 \\
2076 \\
43.67 \\
49.01 \\
56.28 \\
6453 \\
42.63 \\
5.51 \\
16.57 \\
14.27 \\
0.81 \\
257 \\
3.86 \\
2.98 \\
397 \\
0.67 \\
1.52 \\
078 \\
0.18 \\
20.70 \\
142\end{array}$ & $\begin{array}{r}119 \\
124 \\
191 \\
1246 \\
21.7 \\
128 \\
129 \\
83 \\
25.0 \\
20.8 \\
17.5 \\
14.4 \\
13.9 \\
12.1 \\
117 \\
10.8 \\
206 \\
180 \\
227 \\
14.1 \\
130 \\
117 \\
18.0 \\
357 \\
232 \\
138 \\
13.3 \\
214 \\
127 \\
205 \\
15.0 \\
144 \\
213 \\
251 \\
9.9 \\
133 \\
23.9 \\
130 \\
17.1 \\
26.5\end{array}$ & 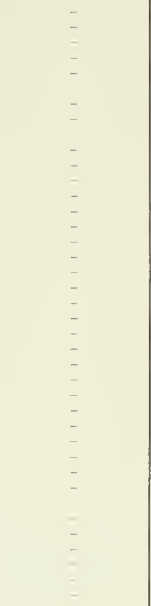 & 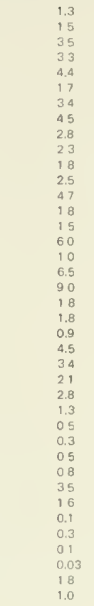 \\
\hline
\end{tabular}


Table 2 Continued

\begin{tabular}{|c|c|c|c|c|c|c|c|c|c|c|c|c|c|c|}
\hline Test Series & Test No." & Object Shape & $\begin{array}{l}\text { Watting Time. } \\
\text { 'w Imint }\end{array}$ & $\begin{array}{l}\text { Waiting Time } \\
\text { Line Foree, } \\
F_{V_{W}}(10)\end{array}$ & $\begin{array}{l}\text { Embedment Depth. } \\
\text { D (tit) }\end{array}$ & $\begin{array}{c}\text { Object Width. } \\
\text { B }(\mathrm{ft})\end{array}$ & $\begin{array}{l}\text { Cross-Sectional } \\
\text { Ares, A }\left(t^{2} t^{2}\right)\end{array}$ & $\begin{array}{l}\text { Displaced Soit } \\
\text { Buoyant Weight. } \\
\text { W }_{5} \text { (tol }\end{array}$ & $\begin{array}{l}\text { Obpect Weight } \\
\text { in Wates. } \\
\text { W } 11 \mathrm{bl} \text {. }\end{array}$ & $\begin{array}{l}\text { 8reakout Lune } \\
\text { Foree. } F_{Q_{b}}(1 b)\end{array}$ & 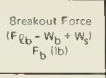 & $\begin{array}{c}\text { Soll Vane } \\
\text { Shear Strength. } \\
\mathrm{S}_{\mathrm{v}}|| \mathrm{lb} / \mathrm{t}^{2} \mid\end{array}$ & 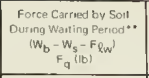 & $\begin{array}{l}\text { Brcakout Time, } \\
\text { Tb tmint }\end{array}$ \\
\hline $\begin{array}{l}\text { FY } 71 \text { laboratory } \\
\text { feuntunued }\end{array}$ & 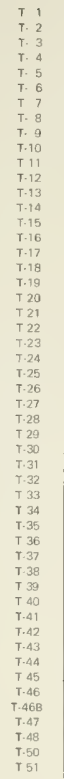 & 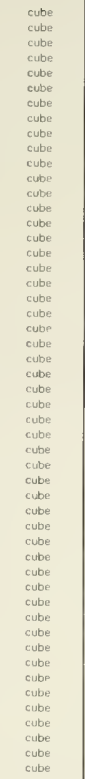 & $\begin{array}{r}3.800 \\
300 \\
225 \\
960 \\
220 \\
150 \\
180 \\
120 \\
1,040 \\
186 \\
90 \\
365 \\
1,200 \\
270 \\
1,140 \\
990 \\
1.365 \\
1,035 \\
1.035 \\
1.365 \\
1.070 \\
1,050 \\
1,080 \\
4.050 \\
4,020 \\
330 \\
286 \\
300 \\
990 \\
270 \\
150 \\
180 \\
180 \\
1,110 \\
1,050 \\
255 \\
210 \\
960 \\
120 \\
90 \\
195 \\
270 \\
165 \\
1,020 \\
1,035 \\
6,940 \\
75 \\
2,580 \\
1,440 \\
240 \\
300\end{array}$ & $\begin{array}{r}1953 \\
10.53 \\
957 \\
9.57 \\
139 \\
1.39 \\
7.57 \\
139 \\
9.02 \\
1.39 \\
20.57 \\
2057 \\
10.07 \\
10.07 \\
22.57 \\
9.57 \\
9.57 \\
1.39 \\
1.39 \\
20.57 \\
1.39 \\
131 \\
139 \\
20.57 \\
9.57 \\
20.57 \\
9.57 \\
139 \\
9.57 \\
1.39 \\
20.57 \\
19.57 \\
1.39 \\
19.57 \\
9.57 \\
1957 \\
9.57 \\
1.39 \\
9.57 \\
1.39 \\
9.57 \\
9.57 \\
1.26 \\
1.43 \\
9.57 \\
20.39 \\
2.57 \\
2.57 \\
1.31 \\
1.31 \\
2.57\end{array}$ & $\begin{array}{l}0.25 \\
0.25 \\
0.25 \\
0.25 \\
0.25 \\
0.25 \\
0.25 \\
0.13 \\
0.25 \\
0.25 \\
0.38 \\
0.25 \\
0.13 \\
0.10 \\
0.25 \\
0.17 \\
0.15 \\
0.08 \\
013 \\
0.38 \\
0.08 \\
0.13 \\
0.08 \\
0.25 \\
0.25 \\
038 \\
0.17 \\
0.13 \\
0.17 \\
008 \\
0.25 \\
0.08 \\
0.13 \\
0.08 \\
0.17 \\
0.08 \\
0.25 \\
0.25 \\
0.17 \\
0.25 \\
0.08 \\
0.02 \\
0.02 \\
0.02 \\
0.02 \\
0.02 \\
0.38 \\
0.38 \\
0.25 \\
0.25 \\
0.50\end{array}$ & $\begin{array}{l}0.75 \\
0.75 \\
0.50 \\
0.50 \\
0.25 \\
0.25 \\
0.50 \\
0.25 \\
0.50 \\
0.25 \\
0.75 \\
0.75 \\
0.50 \\
0.50 \\
0.75 \\
0.50 \\
0.50 \\
0.25 \\
0.25 \\
0.75 \\
0.25 \\
0.25 \\
0.25 \\
0.75 \\
0.50 \\
0.75 \\
0.50 \\
0.25 \\
0.50 \\
0.25 \\
0.75 \\
0.75 \\
0.25 \\
0.75 \\
050 \\
0.75 \\
0.50 \\
0.25 \\
0.50 \\
0.25 \\
0.50 \\
0.50 \\
0.25 \\
0.25 \\
0.50 \\
0.75 \\
075 \\
0.50 \\
0.25 \\
0.25 \\
0.50\end{array}$ & $\begin{array}{l}0.5625 \\
0.5625 \\
0.2500 \\
0.2500 \\
0.0625 \\
0.0625 \\
0.2500 \\
0.0625 \\
0.2500 \\
0.0625 \\
0.5625 \\
0.5625 \\
0.2500 \\
0.2500 \\
0.5625 \\
0.2500 \\
0.2500 \\
0.0625 \\
0.0625 \\
0.5625 \\
0.0625 \\
0.0625 \\
0.0625 \\
0.5825 \\
0.2500 \\
0.5825 \\
0.2500 \\
0.0625 \\
0.2500 \\
0.0625 \\
0.5625 \\
0.5625 \\
0.0625 \\
0.5825 \\
0.2500 \\
0.5625 \\
0.2500 \\
0.0625 \\
0.2500 \\
0.0625 \\
0.2500 \\
0.2500 \\
0.0625 \\
0.0625 \\
0.2500 \\
0.5525 \\
0.5625 \\
0.2500 \\
00625 \\
0.0625 \\
0.2500\end{array}$ & $\begin{array}{l}562 \\
5.62 \\
2.50 \\
2.50 \\
0.63 \\
0.63 \\
250 \\
037 \\
2.50 \\
0.63 \\
8.88 \\
5.62 \\
1.24 \\
0.96 \\
5.62 \\
1.67 \\
150 \\
0.21 \\
0.37 \\
8.88 \\
0.21 \\
0.37 \\
021 \\
5.62 \\
2.50 \\
8.88 \\
1.67 \\
031 \\
1.67 \\
0.27 \\
5.62 \\
187 \\
031 \\
187 \\
1.67 \\
1.87 \\
2.50 \\
0.63 \\
1.67 \\
0.63 \\
0.83 \\
0.211 \\
0.05 \\
0.05 \\
0.21 \\
0.47 \\
8.98 \\
3.75 \\
0.63 \\
0.63 \\
5.00\end{array}$ & 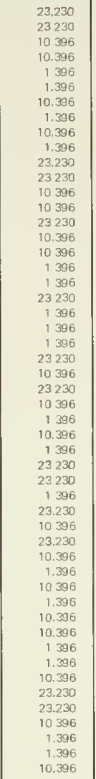 & $\begin{array}{r}29.05 \\
2366 \\
19.61 \\
19.61 \\
3.89 \\
3.89 \\
1427 \\
3.21 \\
25.61 \\
5.49 \\
37.36 \\
42.96 \\
14.20 \\
13.78 \\
28.40 \\
1360 \\
12.48 \\
3.87 \\
196 \\
27.29 \\
2.31 \\
2.44 \\
3.62 \\
38.73 \\
22.26 \\
30.91 \\
17.50 \\
4.58 \\
24.46 \\
5.20 \\
54.76 \\
32.54 \\
3.91 \\
28.76 \\
19.86 \\
35.48 \\
17.80 \\
10.31 \\
14.56 \\
6.56 \\
12.97 \\
10.73 \\
1.52 \\
1.53 \\
10.92 \\
23.80 \\
44.06 \\
21.77 \\
4.33 \\
4.33 \\
2676\end{array}$ & $\begin{array}{r}11.45 \\
16.05 \\
11.72 \\
11.72 \\
3.22 \\
3.22 \\
6.37 \\
2.23 \\
16.71 \\
4.72 \\
22.57 \\
25.36 \\
4.97 \\
4.35 \\
10.79 \\
4.76 \\
3.44 \\
2.58 \\
0.98 \\
12.50 \\
1.23 \\
1.36 \\
2.44 \\
21.13 \\
14.36 \\
16.17 \\
8.66 \\
3.50 \\
16.62 \\
4.02 \\
3716 \\
1119 \\
2.83 \\
7.41 \\
11.02 \\
14.13 \\
9.90 \\
9.54 \\
5.72 \\
5.79 \\
3.41 \\
0.54 \\
0.17 \\
0.19 \\
0.73 \\
1.03 \\
29.17 \\
15.12 \\
3.56 \\
3.56 \\
21.36\end{array}$ & $\begin{array}{l}130 \\
13.2 \\
11.7 \\
14.2 \\
14.5 \\
145 \\
14.2 \\
13.5 \\
24.5 \\
23.4 \\
13.0 \\
22.8 \\
22.0 \\
14.1 \\
22.9 \\
15.7 \\
16.4 \\
21.3 \\
13.2 \\
24.3 \\
13.9 \\
12.4 \\
13.0 \\
16.1 \\
23.8 \\
14.9 \\
211 \\
21.0 \\
20.0 \\
18.5 \\
19.1 \\
14.4 \\
18.2 \\
12.8 \\
20.0 \\
16.9 \\
14.9 \\
20.7 \\
18.0 \\
20.0 \\
22.8 \\
15.3 \\
18.5 \\
13.0 \\
210 \\
23.8 \\
21.9 \\
15.3 \\
21.0 \\
21.0 \\
219 \\
\end{array}$ & $\begin{array}{l}- \\
- \\
- \\
- \\
- \\
- \\
- \\
- \\
- \\
- \\
- \\
- \\
- \\
- \\
- \\
- \\
- \\
- \\
- \\
- \\
- \\
- \\
- \\
- \\
- \\
- \\
- \\
- \\
- \\
- \\
- \\
- \\
- \\
- \\
- \\
- \\
- \\
- \\
- \\
- \\
- \\
- \\
- \\
-\end{array}$ & 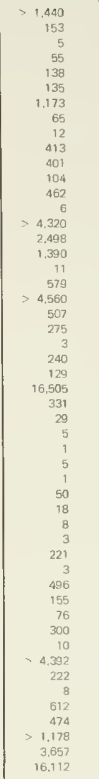 \\
\hline
\end{tabular}

- I indicates immediate break out tests, $T$ indicates long-lerm breakout tests.

. For cases in which bearing capacty is exceeded durino object placement. 
This relationship summarizes the extent to which previous soil mechanics research can be applied to the problem of breakout. To refine the process of predicting immediate breakout forces still further, it is necessary to resort to empirical breakout data. This was done by first selecting a criterion for separating immediate breakout tests from long-term breakout tests. For the field tests and the FY-69 laboratory tests, a breakout time of 10 minutes was set as the division between the two because this time was required for load application. For the FY-71 tests, immediate and long-term tests were conducted differently. The immediate breakout tests were performed by increasing the line load until breakout occurred; whereas the long-term breakout tests were performed by applying a line force equal to some fraction of the estimated immediate force.

The data from the immediate breakout laboratory tests were correlated by plotting the experimentally determined tcrm, $F_{I b} / A S$, versus $D / B$, a technique suggested by the form of Equation 2. This plot is given by Figure 1 .

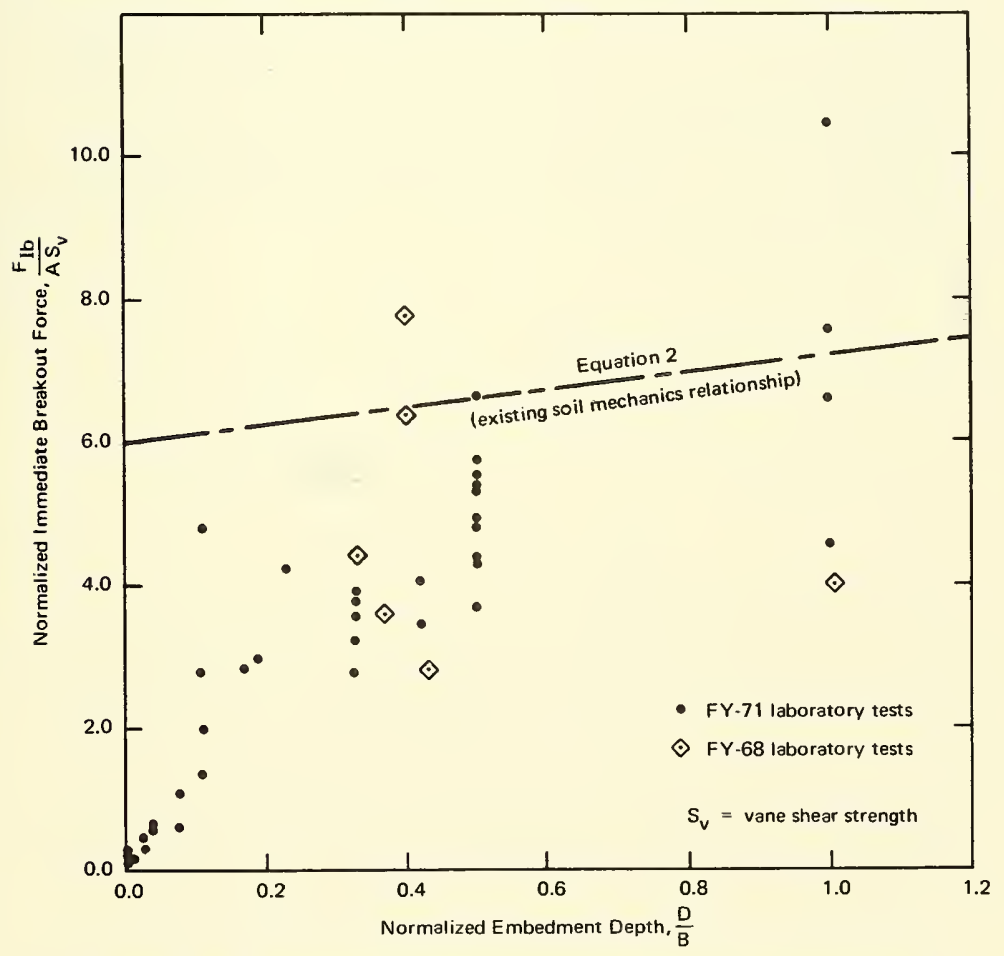

Figure 1. Normalized immediate breakout force versus normalized embedment depth-laboratory tests. 
A slightly different technique was followed for the field tests. It may be explained by considering first that a relationship similar to Equation 2 holds for the bearing capacity of partially embedded objects: ${ }^{10}$

$$
\frac{P-W_{s}}{A S}=6\left(1.0+0.2 \frac{D}{B}\right)
$$

where $P=$ maximum downward force the object is capable of supporting before bearing capacity failure

This equation suggests an in-situ test for measuring the undrained shear strength, S. If an estimate of the buoyant unit weight of the soil is made and the geometry terms A, D, and B are known, the soil strength can be determined by measuring the bearing capacity force, $P$.

The object placement phases of the NCEL field tests can be considered as in-situ tests of this type. Very heavy objects were used, and considerable object penetration occurred during each placement operation. It is indicated that the soil bearing capacity was exceeded at the soil-water interface and that the objects penetrated until the soil strength was adequate to support the object weights. It can be assumed, therefore, that the bearing capacity, $P$, is equal to the object buoyant weight, $W_{b}$, less any line force, $F_{\ell_{w}}$, applied during the waiting period.

Equation 3 can be rewritten as

$$
\frac{W_{b}-F_{l_{W}}-W_{s}}{A S}=6\left(1.0+0.2 \frac{D}{B}\right)
$$

or

$$
\frac{F_{q}}{A S}=6\left(1.0+0.2 \frac{D}{B}\right)
$$

where $F_{\mathrm{q}}=\mathrm{W}_{\mathrm{b}}-\mathrm{F}_{\ell_{\mathrm{w}}}-\mathrm{W}_{\mathrm{s}}$

$=$ force carried by soil during waiting period prior to breakout loading

The field test data were correlated by plotting the experimentally determined term, $F_{1 b} / F_{q}$, versus $D / B$. This plot is presented in Figure 2. 


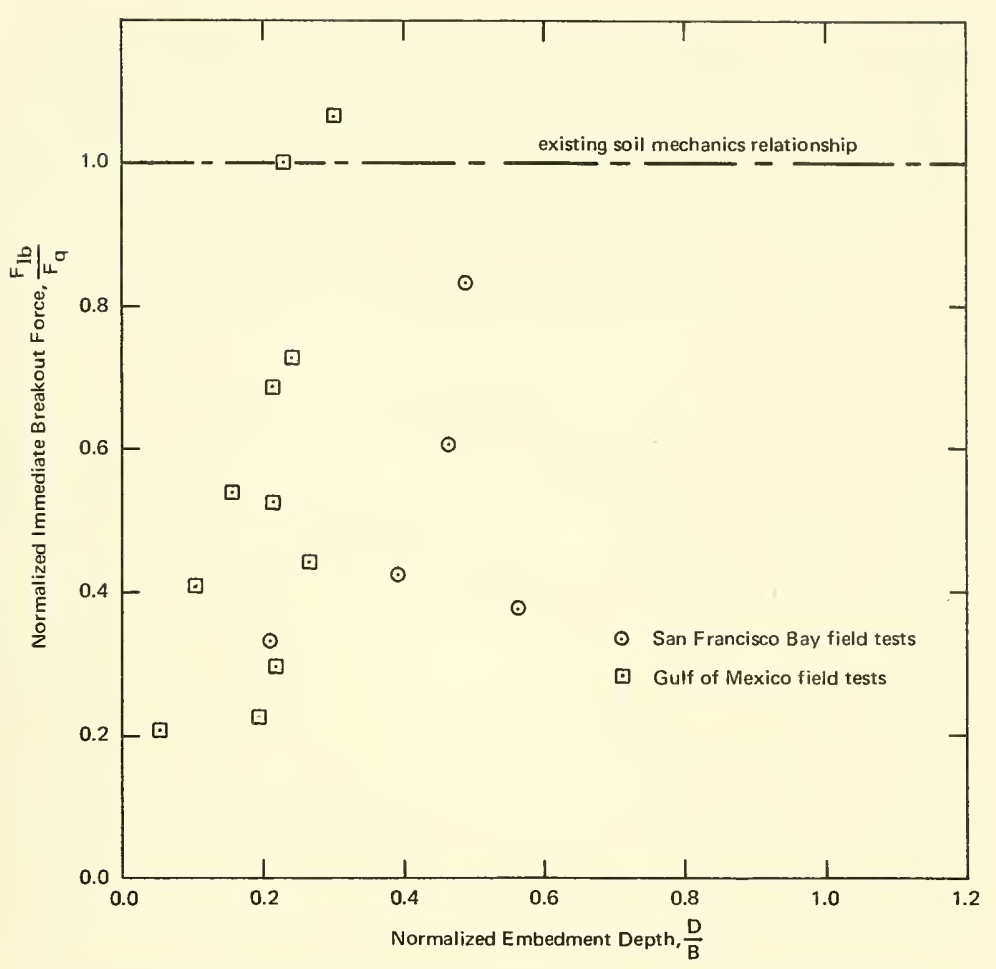

Figure 2. Normalized immediate breakout force versus normalized embedment depth-field tests.

To present all of the immediate breakout data on one plot, it is necessary to convert the breakout force terms for field and laboratory testing into the same form. The form of the laboratory test force terms was altered to that of the field test terms to achieve this objective. The quantity obtained is identified as $F_{\mathrm{Ib}} / F_{\mathrm{q}}^{\prime}$, with $F_{\mathrm{q}}^{\prime}$ equal to $6 \mathrm{AS}[1.0+0.2(\mathrm{D} / \mathrm{B})]$. By referring to Equation 4 , it can be seen that $F_{q}^{\prime}$ is quite similar to $F_{q}$ although the method used to determine it is different. A general plot of $F_{I b} / F_{q}^{\prime}$ and $F_{I b} / F_{q}$ versus $\mathrm{D} / \mathrm{B}$ for all of the immediate breakout tests considered is presented in Figure 3.

By comparing Equations 2 and 4 , it can be seen that $F_{\mathrm{Ib}} / \mathrm{F}_{\mathrm{q}}$ or $\mathrm{F}_{\mathrm{Ib}} / \mathrm{F}_{\mathrm{q}}^{\prime}$ would always equal 1.0 if Equation 2 were strictly correct for breakout. However, as can be seen, there are significant deviations from 1.0 for low values of 
D/B. This deviation may be attributable to elastic deformations which are ignored in the development of bearing capacity equations. When uplift forces are applied to a partially embedded object, the soil-object system will begin to deform upward elastically well before actual rupturing of the soil occurs. If the object is shallowly embedded, these deformations alone might be enough to allow the object to become detached. For deeper embedments, complete rupturing of the soil would be required for breakout.

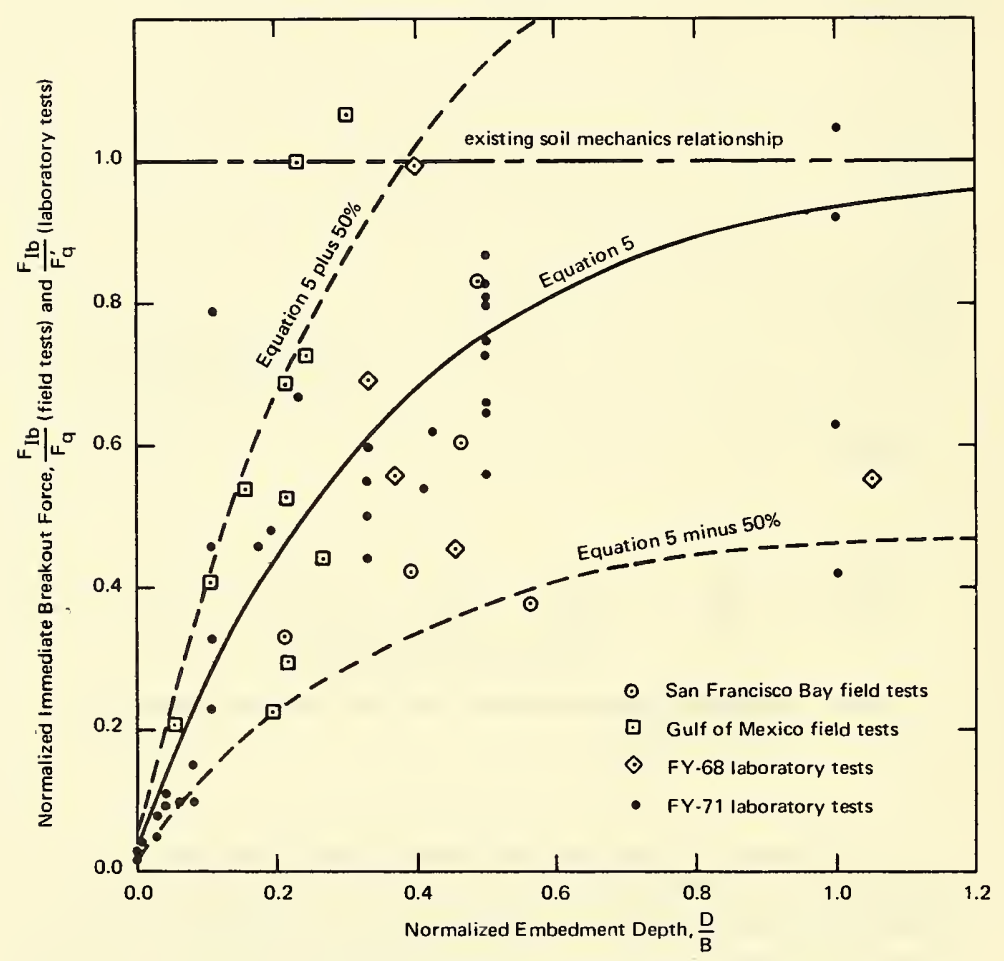

Figure 3. Normalized immediate breakout force versus normalized embedment depth-field and laboratory tests.

The exact reason for the observed behavior is not important. What is important is that all of the immediate breakout force data points appear to fall within a band which can be represented by one equation and can thereby 
provide a basis for accounting for time effects. It is significant that the data are bounded by the upper limit, $F_{\mathrm{Ib}} / F_{\mathrm{q}}$ equal to 1.0, and that a decrease in normalized embedment depth results in a systematic decrease in the normalized breakout force. The trend of the data suggests an exponential form; the coefficients of the appropriate exponential equation were obtained through trial and error in such a manner that the number of points outside the plusor-minus-50\% band was minimized. The resulting empirical equation for predicting the force required for immediate breakout is

$$
\frac{F_{I b}}{F_{q}} \text { or } \frac{F_{I b}}{F_{q}^{\prime}}=1.0-0.97 \mathrm{e}^{-2.75(D / B)}
$$

This equation is plotted in Figure 3 aiong with the lines of plus or minus $50 \%$ of the predicted breakout force. This band includes $88 \%$ of the data points.

A discussion of how to apply Equation 5 practically to predict the required immediate breakout force is given in the SUGGESTED PROCEDURE and SAMPLE PROBLEM sections.

\section{Long-Term Breakout}

Predicting the time required for extracting objects with a force less than the immediate breakout force is somewhat more difficult than predicting the immediate breakout force itself. Existing soil mechanics knowledge provides little information on the subject, and virtually no empirical data exist other than the results of the NCEL tests. A further complication arises from a general lack of knowledge as to the mechanism which leads to breakout under long-term conditions. It is necessary, therefore, to resort to empirical data correlation tempered with observational information on the mechanism to produce a usable prediction technique. Along these lines, the long-term laboratory tests of $\mathrm{FY}-71$ were given first consideration because of their large number, the variety of parameters considered, and the extent of control.

The qualitative characteristics of long-term breakout were determined observationally and by measuring the upward object deflection as a function of time after load application and the depths of the holes left by objects which were broken out. The characteristics determined were:

1. After the application of a line force, $F_{\ell_{\mathbf{b}}}$, less than the estimated immediate breakout line force, $F_{\ell I b}$, but greater than the object weight, a somewhat rapid, upward object motion occurred over a period of about 5 minutes. This initial displacement appeared to vary directly with the force applied relative to the estimated immediate force. 
2. Cracks, surrounding the object and extending out from the object corners, formed during the initial displacement phase. The extent of cracking appeared to vary with the relative force level.

3. The soil near the object moved upward and outward away from the object. The soil farther away from the object moved downward. An elevation view of an object immediately after the initial displacement phase is shown below.

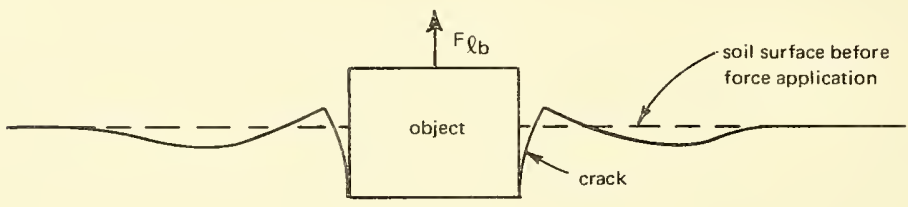

4. After the initial displacement, the object moved upward very slowly until finally becoming detached from the soil. Cracking became more extensive as time progressed.

5. The depth of the hole left after breakout, relative to the initial object embedment depth, varied inversely with the relative force level. In fact, for immediate breakout there was virtually no hole; whereas for very long-term breakout the hole depth approached the original embedment depth.

6. It is uncertain whether or not the object bottom usually became detached from the soil before final breakout. The quantity resulting from subtracting the initial embedment depth from the total upward object displacement before breakout and adding the final hole depth might equal the thickness of a water pocket beneath the object. However, this quantity might also reflect downward elastic deformation after breakout or even erosion caused by water rushing in behind the object during final breakout. This quantity was usually small but greater than zero. In a few of the very long-term tests (for example, 2 weeks), this quantity was virtually zero.

7. The upward displacement immediately preceding final breakout varied directly with the relative force level.

On the basis of these qualitative characteristics, a few tentative conclusions were drawn: 
1. The relative force level appears to be a significant quantity. It will be identified in later sections as $F_{b} / F_{I b}$, with $F_{b}$ equal to the portion of the line force, $F_{\ell_{b}}$, carried by the soil (assumed equal to $F_{\ell_{b}}-W_{b}+W_{s}$ ).

2. The complex process of crack formation and propagation appears to play a major role in determining the breakout response. At least three soil mechanics concepts could enter into this problem:
a. Undrained creep of the soil surrounding the object
b. Drained creep with water flowing into the soil

with time

c. Water flowing through the soil into an expanding cavity beneath the object

Concepts (a) and (b) seem more probable, because they would lead to an expansion of the crack system (as observed), whereas (c) probably would not. An analysis of concepts (b) and (c) would lead to the conclusion that the hydraulic gradient, $\mathrm{p} / \mathrm{D}$, is an important quantity $(\mathrm{p}$ is the gage pressure in the pore water beneath the object and was assumed equal to $F_{b} / A$ ). An analysis of concept (a) would lead to the conclusion that the relative force level by itself is the important quantity in effecting long-term breakout. The importance of these terms can be determined through empirical data correlation.

3. The object embedment depth appears to be very significant in determining breakout.

The significant parameters which were varied among the tests included:

Original embedment depth of object, D

Width of object, B

Hydraulic gradient, $p / D$

Relative force level, $F_{b} / F_{I b}$, with $F_{I b}$ estimated from Equation 5

The dependent variable of all of the long-term breakout tests was the breakout time, $t_{b}$. It was felt that the most important independent variable was the relative force level, $F_{b} / F_{1 b}$. Therefore, the technique followed for determining the best method for correlating data was to plot $F_{b} / F_{I b}$ versus $t_{b}$, normalized by the geometry parameters $D$ and $B$ and the hydraulic gradient $\mathrm{p} / \mathrm{D}$.

Several normalizing procedures were followed. The terms plotted versus $F_{b} / F_{I b}$ included: 


$$
\begin{aligned}
& t_{b} \\
& \frac{t_{b}}{D} \\
& \frac{t_{b}}{D^{2}} \\
& \frac{p t_{b}}{D} \\
& \frac{p t_{b}}{D}\left(\frac{B}{D}\right)^{-} \\
& \frac{p t_{b}}{D}\left(\frac{B}{D}\right)^{2} \\
& \frac{p t_{b}}{D^{2}}\left(\frac{B}{D}\right)^{2}
\end{aligned}
$$

The last term, $\left\langle p t_{b} / D^{2}\right)(B / D)^{2}$, produced the best correlation with $\mathrm{F}_{\mathrm{b}} / \mathrm{F}_{\mathrm{Ib}}$ for the FY-71 laboratory tests. A plot of this relationship is presented in Figure 4. The dimensions of this normalized time term are time-force per length to the fourth power. This term can be nondimensionalized by multiplying by a permeability and dividing by the unit weight of water. Such a nondimensional term can be rationalized as discussed in Appendix C.

The field and FY-68 laboratory test data were also plotted in the form of $F_{b} / F_{I b}$ versus $\left(p t_{b} / D^{2}\right)(B / D)^{2}$, as shown in Figure 5 . Here the correlation is somewhat weaker and the points tend to lie to the left of the data points for the FY-71 laboratory tests (Figure 4). The shift in the data points appears attributable to a variation in some time-related soil property (possibly permeability) among the different soils tested. It is felt that if this property were measured accurately it could be incorporated into the normalized time term (as discussed in Appendix $\mathrm{C}$ ) to improve the quality of the correlation. The permeability of the FY-71 laboratory test soil was measured $10.9 \times$ $\left.10^{-6} \mathrm{~cm} / \mathrm{sec}\right)$, as was the vertical permeability of core samples obtained at the Gulf of Mexico test site $\left(1.1 \times 10^{-7} \mathrm{~cm} / \mathrm{sec}\right)$. The latter measurements, however, are unrepresentative of the overall permeability characteristics of that site because the horizontal permeability was not determined. Because sand seams were observed in the cores, horizontal seepage would assuredly dominate the long-term soil response. Therefore, a more accurate normalization of the data is not possible at present. 


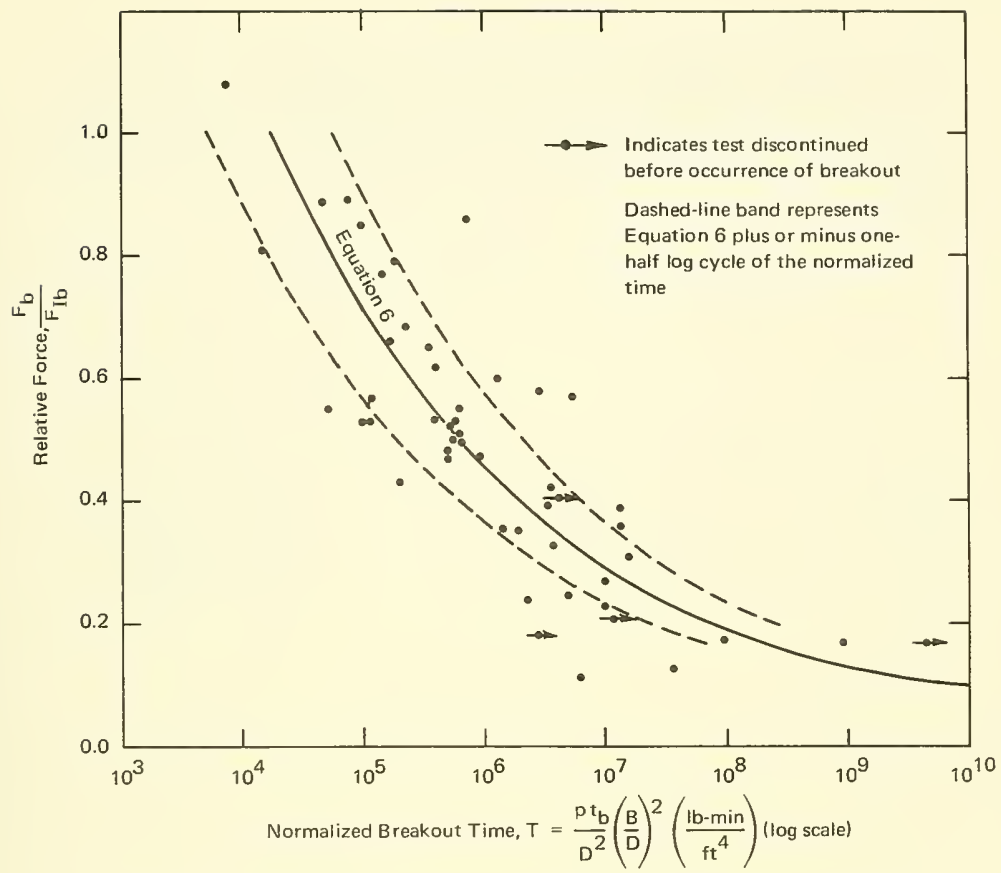

Figure 4. Relative force versus normalized breakout time-FY-71 long-term laboratory tests.

It is felt, however, that the present conditions are sufficiently accurate for estimating the order of magnitude of breakout time. The Gulf of Mexico and San Francisco Bay soils are representative of typical deep-sea deposits, so correlations based on these data can be extrapolated to other sites. The FY-71 laboratory data have a high enough correlation to provide a good indication of the proper form of the breakout time relationship.

The procedure followed was to fit an equation to the FY-71 test data accurately and then shift this equation slightly by altering one parameter to fit the field test data. The test data suggested a log-log form; therefore, a relationship for the FY.71 data was determined to be

$$
\log _{10} \frac{F_{b}}{F_{I b}}=-0.193\left(\log _{10} T-4.24\right)
$$

where $T=\left(p t_{b} / D^{2}\right)(B / D)^{2}$ 
This relationship was obtained by trial and error to minimize the points falling outside the band of the relationship plus or minus one-half log cycle of T. Equation 6 is plotted in Figure 4 along with the band designating plus or minus one-half $\log$ cycle of $\mathrm{T}$. This band contains $70 \%$ of the data points.

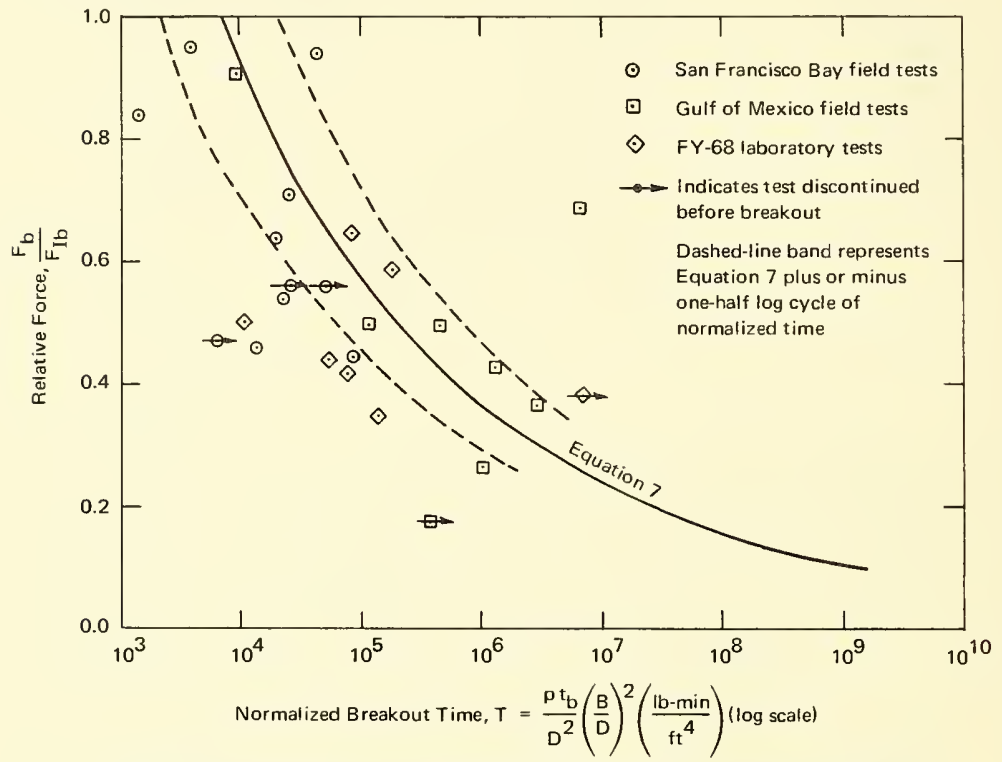

Figure 5. Relative force versus normalized breakout time-long-term field tests and FY-68 long-term laboratory tests.

By using Equation 6 as a guide, a similar relationship was developed for the field test data:

$$
\log _{10} \frac{F_{b}}{F_{I b}}=-0.193\left(\log _{10} T-3.84\right)
$$

The same criterion was used to obtain this equation as was used for Equation 6. Equation 7 is plotted in Figure 5 along with the band designating plus or minus one-half $\log$ cycle of $T$. This band contains $53 \%$ of the data points. Only $13 \%$ of the points fall to the right (unconservative) side of the band. 
Because it reflects the response of seafloor soils in situ, Equation 7 is suggested for predicting the time required for the breakout of partially embedded objects from cohesive seafloor soils in field operations. It is suggested because it reflects the appropriate permeability conditions, has the form indicated by the more rigorously controlled laboratory studies, and, in any case, does not differ significantly from Equation 6. A more complete discussion of how to apply it is included in the SUGGESTED PROCEDURE section.

\section{SUGGESTED PROCEDURE}

The procedure suggested for determining the effort required to achieve unaided breakout of partially embedded objects is shown graphically by the block diagram of Figure 6 and described by the following outline. The letternumber designations included in the blocks refer to sections of the outline. It is assumed that the object has been located and that its geometry is known or can be measured.

A. Measure object geometry parameters A, B, and L; embedment depth, D; and buoyant weight, $W_{b}$.

1. $A$ is the horizontal cross-sectional area of the object at the soil-water interface.

2. $\mathrm{B}$ is the least lateral dimension (width) at the soil-water interface.

3. $L$ is the largest lateral dimension (length) at the soil-water interface.

4. $D$ is the embedment depth. It is assumed equal to $V_{s} / A$, where $V_{s}$ is the volume of that portion of the object embedded beneath the soil-water interface.

B. Determine whether this is a partially embedded object.

1. Calculate $\mathrm{D} / \mathrm{B}$. If it is greater than 1.0, embedment is not considered partial; and anchor-type breakout equations must be used. ${ }^{1,2}$

2. If $\mathrm{D} / \mathrm{B}$ is less than or equal to 1.0 , the problem is considered to be one of partial embedment and the relationships of this report can be used.

C. Determine the general type of seafloor soil involved.

1. This can be accomplished either observationally from a submersible or by obtaining a disturbed, grab sample. 
2. If the soil is cohesionless (sand), breakout will not be a problem. Breakout can be accomplished by applying a line force equal to the buoyant weight of the object. A short time may elapse before the object breaks free.

3. If the soil is cohesive (clay), the soil will provide at least some resistance to breakout, as indicated by the relationships of this report.

D. Estimate the buoyant unit weight of the soil, $\boldsymbol{\gamma}_{\mathrm{s}}$.

1. This can be obtained accurately from a bulk wet density test on a core sample.

2. If core samples are not available, the buoyant unit weight can be taken as 30 pcf, a typical value for seafloor soils.

E. Determine, if possible, how the object became embedded.

1. If the object was sinking slowly (less than or equal to $2 \mathrm{fps}$ ) when it collided with the seafloor, and the present relative embedment depth, D/B is greater than 0.25 , it is not necessary to measure or estimate the soil strength. Instead, the following procedure should be used:

a. Calculate $F_{q}=W_{b}-W_{s}$, where $W_{s}=\gamma_{s} V_{s}$.

b. Refer to Figure 3 (or insert $D / B$ into Equation 5) and obtain the quantity $\mathrm{F}_{\mathrm{Ib}} / \mathrm{F}_{\mathrm{q}}$.

c. Multiply $F_{\mathrm{Ib}} / F_{\mathrm{q}}$ by $F_{\mathrm{q}}$ to obtain $F_{\mathrm{Ib}}$, the portion of the immediate breakout force which will be carried by the soil.

2. If the object penetrated the seafloor with a velocity greater than $2 \mathrm{fps}$, or if the present relative embedment depth, D/B, is less than 0.25 , it is necessary to estimate the undrained shear strength of the soil.

a. This can be accomplished by in-situ vane tests near the object, laboratory vane tests on core samples, or, if neither of these courses can be followed, by reference to Figure 7. This figure presents a typical strength profile for a cohesive seafloor soil.

b. Once the shear strength profile is known, it is necessary to select a representative shear strength. This can be done by averaging the strength from a sediment depth of 0 to $D+B$. 


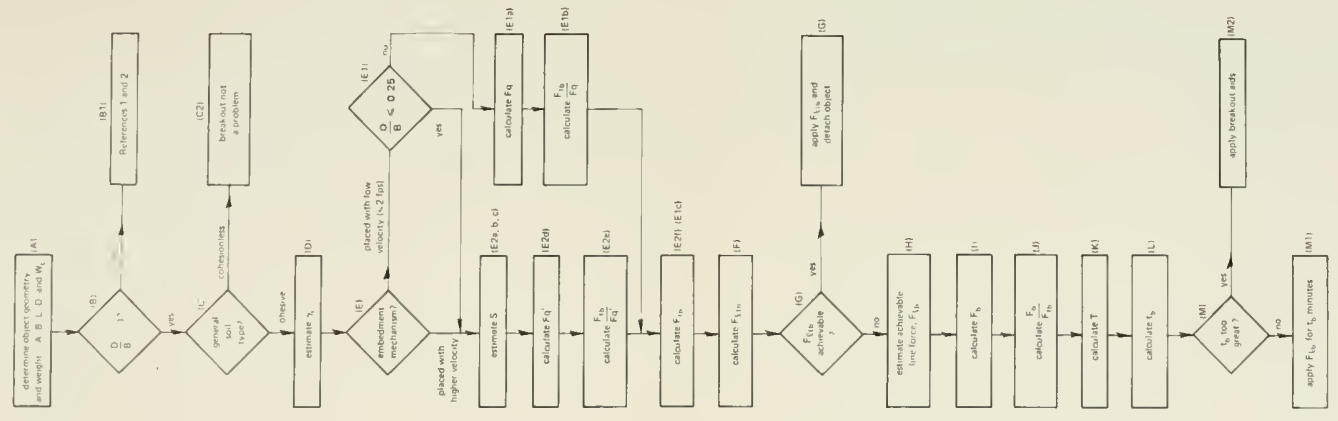



C. The average strength is identified as $\mathbf{S}$.

d. Insert A, S, D, L, and B into the following relationship and solve for $F_{q}^{\prime}$.

$$
F_{q}^{\prime}=5 \mathrm{AS}\left(1.0+0.2 \frac{\mathrm{D}}{\mathrm{B}}\right)\left(1.0+0.2 \frac{\mathrm{B}}{\mathrm{L}}\right)
$$

e. Insert $\mathrm{D} / \mathrm{B}$ into Equation 5 (or refer to Figure 3) and calculate $F_{I \mathbf{I b}} / F_{\mathrm{q}}^{\prime}$.

f. Multiply $F_{I b} / F_{q}^{\prime}$ by $F_{q}^{\prime}$ to obtain $F_{I b}$, the portion of the immediate breakout force which will be carried by the soil.

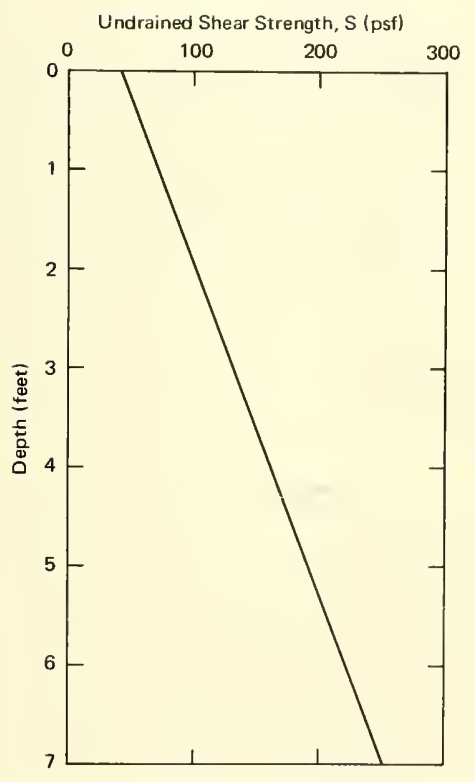

Figure 7. Typical undrained shear strength profile for cohesive seafloor soils.
F. Calculate the line force required for immediate breakout, $F_{\ell \text { Ib }}$.

$\mathrm{F}_{\ell \mathrm{Ib}}=1.5 \mathrm{~F}_{\mathrm{Ib}}+\mathrm{W}_{\mathrm{b}}-\mathrm{W}_{\mathrm{s}}$

A safety factor of 1.5 on $F_{I b}$ is included to allow for the scatter of data in Figure 13.

G. Determine whether or not $F_{\ell \mathrm{Ib}}$ can be applied with the equipment available. If it can, apply $\mathrm{F}_{\text {QI }}$ and detach the object. If it cannot, proceed with the following steps.

$H$. Determine what line force can be achieved and identify it as $\mathbf{F}_{\ell_{\mathbf{b}}}$.

I. Calculate $F_{b}=F_{\ell b}-W_{b}+W_{s}$.

J. Calculate $F_{b} / F_{I b}$, with $F_{I b}$ as calculated in either step E1c or step E2f.

K. Refer to Figure 5 (or Equation 7) and obtain $\mathrm{T}$.

L. Calculate the time required for breakout, $t_{b}$.

$$
t_{b}=\frac{T D^{2}}{p}\left(\frac{D}{B}\right)^{2}
$$

where $p=F_{b} / A$ (foot/pound/ minute units must be used). 
M. After applying a safety factor of 2.0 to $t_{b}$, determine whether the load $\boldsymbol{F}_{\ell_{\mathbf{b}}}$ can be applied economically for this period of time.

1. If it can, apply $F_{\ell_{b}}$ for $t_{b}$ minutes.

2. If it cannot, a system of breakout aids must be devised. 3,4

\section{SAMPLE PROBLEM}

An example of an application of the suggested prediction procedure follows. The step identifications are identical to those of the preceding section.

\section{Problem}

A cylindrical object weighing 50,000 pounds in water is embedded in the seafloor. A grab sample indicates that the seafloor soil is a cohesive material. A submersible visiting the site reports that the axis of the cylinder is parallel to the seafloor and that the cylinder is embedded to its center. The cylinder is 15 feet long and 6 feet in diameter. It has been determined that the object was drifting downward very slowly when it collided with the seafloor. The effort required to detach the object is to be predicted.

\section{Solution}

A. 1. $A=15 \times 6=90 \mathrm{ft}^{2}$

2. $B=6 \mathrm{ft}$

3. $L=15 \mathrm{ft}$

4. $V_{s}=\pi \times 3^{2} \times 15 \times 0.5=212 \mathrm{ft}^{3}$

$$
D=V_{s} / A=212 / 90=2.36 \mathrm{ft}
$$

$W_{\mathrm{b}}=50,000 \mathrm{lb}$

B. $D / B=0.393$. Therefore, the object is partially embedded.

C. The soil is cohesive.

D. Assume $\gamma_{\mathrm{s}}=30$ pcf.

E. The object was sinking slowly and the relative embedment depth is greater than 0.25 . Therefore, the shear strength does not need to be estimated. 


$$
\begin{aligned}
& \text { 1. a. } F_{\mathrm{q}}=\mathrm{W}_{\mathrm{b}}-\mathrm{W}_{\mathrm{s}} \\
& W_{s}=\gamma_{s} V_{s}=30 \times 212=6,360 \mathrm{lb} \\
& W_{b}=50,000 \mathrm{lb} \\
& F_{\mathrm{q}}=50,000-6,360=43,640 \mathrm{lb} \\
& \text { b. } D / B=0.393 \\
& \text { from Figure } 3 \text { (or Equation 5) } \\
& \mathrm{F}_{\mathrm{Ib}} / \mathrm{F}_{\mathrm{q}}=0.670 \\
& \text { c. } F_{\mathrm{Ib}}=0.670 \times 43,640=29,240 \mathrm{lb} \\
& \text { F. } F_{\ell \mathrm{Ib}}=F_{\mathrm{Ib}}+W_{\mathrm{b}}-W_{\mathrm{s}}=29,240+50,000-6,360 \\
& =72,880 \mathrm{lb}
\end{aligned}
$$

or, with a safety factor of 1.5 ,

$$
F_{\ell \mathrm{lb}}=(1.5 \times 29,240)+50,000-6,360=87,500 \mathrm{lb}
$$

To ensure immediate breakout, a line force of 87,500 pounds should be applied to the object.

G. It is determined that a force of 87,500 pounds cannot be applied with the existing equipment.

H. A force of 60,000 pounds, however, can be applied. The amount of time required for breakout with this force is desired.

I. $F_{b}=F_{l_{b}}-W_{b}+W_{s}$

$F_{\ell_{b}}=60,000 \mathrm{lb}$

$$
F_{b}=60,000-50,000+6,360=16,360 \mathrm{lb}
$$

J. $F_{b} / F_{\text {Ib }}=16,360 / 29,240=0.56$

K. From Figure 5 (or Equation 7)

$$
\begin{aligned}
T & =1.41 \times 10^{5}\left(\mathrm{lb}-\mathrm{min} / \mathrm{ft}^{4}\right) \\
t_{b} & =\frac{T D^{2}}{p}\left(\frac{D}{B}\right)^{2} \\
p & =\frac{F_{b}}{A}=\frac{16,360}{90}=182 \mathrm{psf} \\
t_{b} & =\frac{1.41 \times 10^{5} \times(2.36)^{2}}{182}\left(\frac{2.36}{6}\right)^{2}=6.7 \times 10^{2} \mathrm{~min} \\
& =11.1 \mathrm{hr}
\end{aligned}
$$


Application of a safety factor of 2 yields a design breakout time of 22 hours. This might be a reasonable time to wait for breakout to occur. If not, breakout aids should be applied.

\section{SUMMARY AND CONCLUSIONS}

1. Breakout of partially embedded objects from cohesive seafloor soils can be divided into immediate and long-term breakout. Immediate breakout involves rapidly increasing the upward force on an embedded object until it becomes detached. Long-term breakout involves applying a force less than the immediate breakout force and allowing time to pass. If embedment is partial (embedment depth less than object width) breakout will eventually occur under the influence of almost any net uplift force. However, much time may be involved.

2. The immediate breakout force problem appears similar to the problem of determining the bearing capacity of shallow footings. However, the basic equations must be modified somewhat to reflect differences between failure mechanisms.

3. The techniques of this report are capable of predicting the immediate breakout force (that is, that portion in addition to the object weight) within plus or minus $50 \%$. This is a suitable accuracy for a seafloor object retrieval operation.

4. The breakout forces required for objects very shallowly embedded (embedment depth less than one-tenth the object width) are almost negligible.

5. No existing theoretical techniques appear applicable to the long-term breakout problem. However, the empirical approach presented in this report provides a means for predicting the breakout time within about plus or minus $100 \%$. This is a reasonable accuracy for a time-dependent soil mechanics problem and appears adequate for the types of operations toward which this approach will be applied.

\section{RECOMMENDATIONS}

1. The procedures presented in this report should be used to predict the immediate breakout force and the time required for breakout when a force less than the immediate breakout force is applied. 
2. Although these procedures are approximate, additional research in the area of partially embedded object breakout is not justified at present. The accuracies achievable appear adequate for the sorts of operations toward which these procedures will be applied. These accuracies are considerably greater than those achievable for deeply embedded object breakout and aided breakout.

3. Future research should be directed toward developing techniques, similar to those of this report, for predicting the effort required for deeply embedded object breakout and aided breakout.

4. Extensive small-scale model laboratory studies, supplemented by limited field tests, appear to offer the most economical course for this research. 
Appendix A

FY-71 LABORATORY TESTS

\section{GENERAL}

Small-scale model immediate and long-term breakout tests were performed in the NCEL Seafloor Soils Laboratory in FY-71. The tests were initiated specifically to investigate the breakout of objects of very shallow embedment for application to the various NCEL programs which involve bottom-sitting devices. In addition, however, a number of tests with somewhat greater embedment depths were performed in order to allow comparison with earlier NCEL tests and to provide additional, well-controlled test data on the general problem of partially embedded object breakout.

In all, about 90 tests were performed. Of these, 40 were classed as immediate breakout tests, in which upward loads on embedded objects were increased rapidly to breakout. The remainder were long-term breakout tests. A fraction of the immediate force was applied and the time required for breakout was measured.

\section{EQUIPMENT}

Simple experimental equipment was used so that a large number of tests could be performed economically. The equipment consisted of two 55-gallon oil drums on which were mounted knife-edges for use as fulcrums to levers. The levers were solid steel bars, 4 feet long, with mercury switches mounted near their centers. The drums were partially filled with a cohesive soil into which objects were embedded. Lines were attached from the objects to the inner ends of the levers. At the other ends of the levers were placed weight stands on which weights were added to provide the required upward line forces to the embedded objects. The mercury switches were wired into circuits containing electric clocks. When breakout occurred and the levers deflected, the mercury switches opened, thereby stopping the clocks. It was not necessary, therefore, to observe the tests continuously. A diagram of the testing configuration is shown in Figure A-1.

It was felt that the soil shear strength would become a critical parameter during the data analysis phase. Therefore, special attention was given to developing a simple and accurate device for measuring the in-situ strength of the soil within the drums. A hand-held device was developed which consisted of a 1.4inch-diameter, 1.4-inch-long, four-bladed vane mounted on a 24-inch aluminum 
shaft which was in turn connected to a torque screwdriver handle. The vane size was selected so that typical soil strengths would fall within the midrange of the torque dial. The shaft without the vane was tested to determine the torque lost to shaft friction. The torque device was calibrated by applying a known torque and comparing with the dial reading.

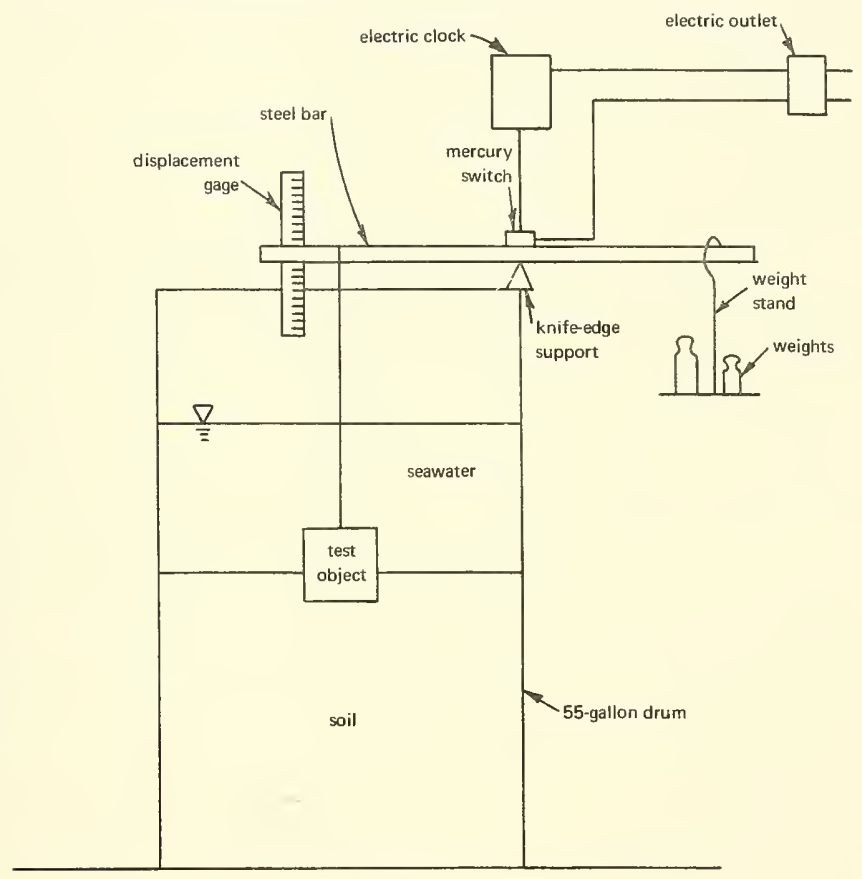

Figure A-1. Setup for FY-71 laboratory tests.

Vane tests were performed by pressing the vane to a particular soil depth and then slowly twisting the handle until a constant reading was obtained. To achieve as much consistency as possible, the same individual performed all tests. A few core samples of the soil were obtained and subjected to the more standard laboratory vane test. The results of these latter tests compared favorably with the results of the tests with the hand-held vane. Strengths were obtained according to the usual practice of assuming full mobilization of strength over a cylindrical failure surface having a diameter equal to the vane diameter and an altitude equal to the vane height. Mobilization of strength along the ends of the cylinder was also assumed. 
The upward movement of objects during long-term breakout was monitored with a simple deflection gage. The movement of the inner end of the lever relative to the side of the 55-gallon drum was measured.

Three objects were used in the testing: a $3 \times 3 \times 3$-inch block, a $6 \times 6 \times 6$-inch block, and a $9 \times 9 \times 6$-inch block. The blocks were concrete, and their weights in seawater were: $1.39,10.39$, and 23.23 pounds, respectively. The $9 \times 9 \times 6$-inch block was always embedded with the $9 \times 9$-inch plane horizontal.

\section{TEST SOIL}

The soil tested was a marine, clayey silt obtained from a tidal flat at Seal Beach Naval Weapons Station, California. The soil was oven-dried, broken into particles, and mixed with saltwater to obtain desired strengths. Some of the soil index properties are given in Table A-1.

Table A-1. Properties of Typical Soil Sample-FY-71 Laboratory Tests

\begin{tabular}{|l|c|}
\hline \multicolumn{1}{|c|}{ Property } & Value or Description \\
\hline Bulk wet density (pcf) & 104 \\
Vane shear strength (psf) & 10 \\
Remolded strength (psf) & 5 \\
Sensitivity & 2 \\
Water content (\%) & 57 \\
Specific gravity of solids & 2.60 \\
Void ratio & 1.45 \\
Liquid limit (\%) & 41 \\
Plastic limit (\%) & 26 \\
Plasticity index (\%) & 15 \\
Sand (\%) & 10 \\
Silt (\%) & 58 \\
Clay (\%) & 32 \\
Classification (Unified Soil Classification System) & ML (slightly plastic clayey silt) \\
\hline
\end{tabular}




\section{TEST PROCEDURE AND RESULTS}

A list of the tests which would be performed was compiled before the testing program was begun. It was decided that the parameters to be varied would be the soil strength, $S$; the embedment depth, D; the object width, $B$; and the relative breakout force, $F_{b} / F_{I b}$ (for the long-term tests). To be investigated were two soil strengths (10 and 20 psf), three object widths $(3,6$, and 9 inches), and a variety of embedment depths (ranging between 0 and 4.5 inches). Relative force levels ranging between 0.2 and 1.0 were also to be considered. A list of the various permutations of these parameters was made and randomized. Two tests with each permutation were eventually performed. A few additional tests were performed when it appeared that data for a particular range of parameter values were lacking.

Each test was begun by thoroughly mixing the soil in one of the two drums. Either additional dry soil or water was added until the desired shear strength was obtained throughout the drum. The soil was then vibrated to increase the degree of saturation. An object was placed to a predetermined embedment depth and the soil smoothed about it. About 1 foot of water was added to cover the soil and object, and the lever-weight-holder system was rigged. Weights were added so that the weight of the object was supported and virtually no force, positive or negative, was transmitted to the soil. This condition was maintained for about 24 hours.

At the end of the waiting period, additional weights were placed on the weight hanger. If the test was to be an immediate breakout test, weights were placed until breakout occurred. For a long-term test, a predetermined weight was placed on the holder, and the time required for breakout was recorded with the electric-clock-mercury-switch system. The upward movement of the object was measured as a function of time after load application.

After a test was completed, the water was drained from the drum, and the characteristics of the hole left by the object were measured. The shear strength of the soil was then measured at four different depths (1, 4, 7 , and 13 inches) beneath the hole center and at the same four depths at an offset distance of 8 inches from the hole center. For use in analysis, these were averaged as follows:

(a) With the $3 \times 3 \times 3$-inch block, the strengths at depths of 1 and 4 inches and offset distances of 0 and 8 inches from the hole center were averaged to yield one shear strength, $\mathrm{S}$.

(b) With the $6 \times 6 \times 6$-inch and $9 \times 9 \times 6$-inch blocks, the strengths at depths of 1,4 , and 7 inches at the two different offset distances were averaged.

The data from these tests are included in Table 2 of the main text of this report. 


\section{Appendix B}

\section{BEARING-CAPACITY ANALOGY}

One of the classic problems considered in soil mechanics is the determination of the bearing capacity of a shallow footing on an infinite half-space. The bearing capacity is defined as the maximum downward load which may be applied to the footing before the underlying soil is ruptured along continuous failure surfaces in such a way as to create a failure mechanism. This condition is also often referred to as a general shear failure or a state of plastic equilibrium. The usual procedure used to analyze this situation has been to construct a series of hypothetical failure surfaces, assign to each soil element along the surfaces a shear stress equal to the soil strength, and solve the resulting structure by statics to obtain the footing load. Theoretically. if this were done for every imaginable series of surfaces, the bearing capacity would correspond to the lowest footing load obtained. A substantial effort has been directed toward finding appropriate failure surfaces and has led to the development of several bearing-capacity equations. One of the most common of these is that developed by Prandtl for an infinitely long footing of finite width; this equation is based on the failure pattern shown in Figure B-1. ${ }^{11}$

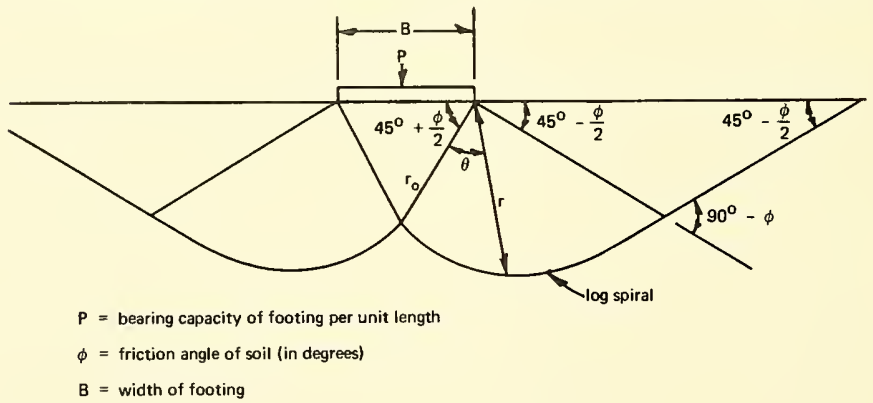

The log spiral is given by the equation

$$
\begin{aligned}
& r=r_{0} e^{\theta \tan \phi} \\
& r, r_{0} \text {, and } \theta \text { are defined in the diagram ( } \theta \text { is measured in radians) } \\
& r_{0} \text { is the length of one of the sides of the isosceles triangle }
\end{aligned}
$$

Figure B-1. Cross section illustrating PrandtI's plastic equilibrium theory. 
It can be seen from Figure B-1 that the characteristics of PrandtI's failure surfaces are strongly related to the soil friction angle. For short-term loadings on fine-grained, low-permeability (cohesive) soils such as those usually found on the seafloor, the assumption is often made that the apparent friction angle is equal to zero. This is because the low soil permeability prevents significant drainage and soil volume change during a short-term loading. Because soil strength is primarily a function of relative volume (void ratio), there will be little strength variation, and the soil friction angle, which is basically a measure of the change in strength corresponding to a change in stress, will appear to be zero. ${ }^{12}$ For a soil with zero friction angle, Prandtl's failure surfaces reduce to those shown in Figure B-2.

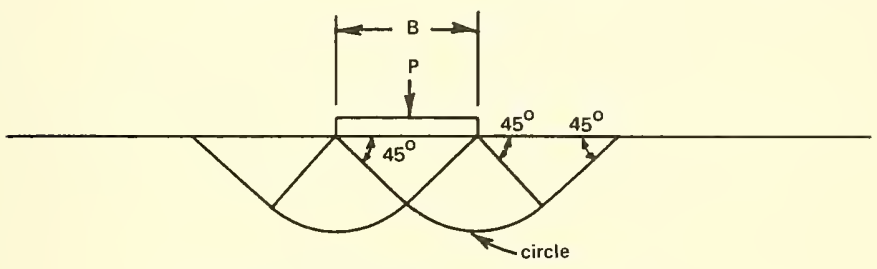

Figure B-2. PrandtI's failure surfaces with $\phi=0$.

If it is assumed that the shear stresses along all of the failure surfaces are identically equal to the undrained soil strength, $\mathrm{S}$, this problem can be solved by balancing moments to yield the equation

$$
P=2 B\left(\frac{\pi}{2}+1\right) S=5.14 B S
$$

At this point, it should be noted that the failure surfaces of Figure B-2 are symmetric about the footing edges. Because the statics problem is solved by balancing moments about these edges, it can be seen that the solution to the problem shown in Figure B-3 is identical to that given for Figure B-2.

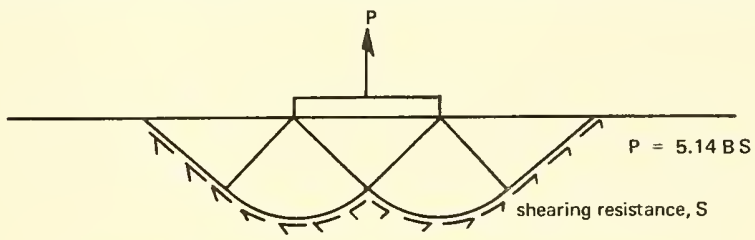

Figure B-3. Prandtl's failure surfaces with upward loading. 
Figure B-3 represents a simple breakout problem involving the following mechanism: an upward load per unit length, $\mathrm{P}$, is applied to a long, narrow footing. Because loading is rapid (immediate breakout), the soil permeability is low, and there are no open passageways, water is prevented from moving into the zone beneath the footing. Instead the soil itself is forced to move into this zone and the flow pattern of the soil is given by the Prandtl failure surfaces.

The situation involved in the NCEL breakout tests was somewhat different from that given by Figure B-3. The objects were not long, narrow footings but rather were cubes, spheres, and prisms; and they were partially embedded in the subsoil. The downward short-term bearing capacity for objects in this situation has been investigated by Skempton, ${ }^{10}$ who presented the equation

$$
\frac{P}{A}=5 S\left(1.0+0.2 \frac{D}{B}\right)\left(1.0+0.2 \frac{B}{L}\right)+\frac{V_{s} \gamma_{s}}{A}
$$

where $\mathbf{P}=$ bearing capacity force

$$
\begin{aligned}
& A=\text { horizontal cross-sectional area of object } \\
& S=\text { undrained shear strength of soil } \\
& D=\text { depth of embedment } \\
& B=\text { width of object } \\
& L=\text { length of object } \\
& V_{S}=\text { volume of object embedded in soil } \\
& \gamma_{S}=\text { buoyant unit weight of soil }
\end{aligned}
$$

This equation is somewhat empirical; but it does reduce approximately to the solution of Prandtl's equation for a long, narrow footing with zero embedment depth, and it has been shown to be applicable to actual field situations. In view of the similarity between breakout and bearing capacity as noted in the simpler Prandtl example, it is reasonable to assume that this equation will also yield an approximate solution to the breakout problem for these more complex circumstances. The comparison between the two is probably not exact for this case because of some distortion of the symmetric failure surfaces and differences in elastic deformation patterns. However, an equation of this sort can serve as a rough first approximation in estimating the immediate breakout force. 
One difference between the two approaches should be corrected. In Equation B-2, the last term, $V_{s} \gamma_{s} / A$, represents a buoyancy effect; the deeper an object is embedded, the greater will be the force required to cause a bearing capacity failure because of the tendency of the surrounding soil to buoy the object upward. In breakout, because this buoyancy effect will cause a reduction in the required uplift force, the final term should be subtracted rather than added. This effect may also be incorporated by including the buoyancy effect in the breakout force term. The resulting breakout force equation, as patterned after Skempton's bearing capacity equation, is

$$
\frac{F_{\text {Ib }}}{A}=5 S\left(1.0+0.2 \frac{D}{B}\right)\left(1.0+0.2 \frac{B}{L}\right)
$$

where $\mathbf{F}_{\mathrm{Ib}}=$ portion of immediate breakout line force, $\mathrm{F}_{\mathbf{\ell} \mathrm{Ib}}$, carried by soil

$$
\begin{aligned}
& =F_{\ell I b}-W_{b}+W_{s} \\
W_{b} & =\text { buoyant weight of object } \\
W_{s} & =\text { buoyant weight of soil displaced by object } \\
& =V_{s} \gamma_{s}
\end{aligned}
$$

The limiting equilibrium problem could also be approached by formulating the general equations of elasticity and plasticity numerically and solving them on the computer. This has been done for the bearing capacity of an unembedded strip footing, and the results obtained were found to agree well with Prandt/'s equation. ${ }^{13}$ The investigation was valuable in that it illustrated how plastic zones develop in soil before the bearing capacity is reached. It did not, however, lead to the development of a new bearing capacity equation.

The numerical approach has also been applied to the breakout problem. ${ }^{6}$ Once again, the results of the computer program written for the problem yield information on how plastic zones develop during shear. However, in terms of predicting the required ultimate breakout force, there is little difference between this approach and that summarized by Equation B-3. In the sample problem included in Reference 6, the breakout force required to extract a particular submarine from a specific seafloor site was predicted to be 91,000 pounds. By use of the same input parameters, Equation B-3 predicts a breakout force of 86,500 pounds.

In comparing these results, it should be noted that the numerical solution of Reference 6 required considerable computer time to process and that the computer program required as input the soil elastic modulus and 
Poisson's ratio as well as undrained shear strength. Also, it should be noted that in the sample problem of Reference 6 , a Poisson's ratio of 0.4 was assumed for the soil. A value of 0.5 would have been more appropriate for undrained, short-term breakout so a significant portion of the difference between the two predictions may be attributable to the Poisson's ratio assumption rather than to any basic analytic discrepancies.

Considering the complexity of the breakout problem, particularly with regard to time effects, there is little justification for undue sophistication in formulating the initial limiting equilibrium relations. Modifications will have to be made to any relationship to account for actual field effects. It is much simpler to modify a relationship such as Equation B-3 than it is to modify an involved numerical analysis. 


\section{Appendix C}

\section{DISCUSSION OF FORM OF NORMALIZED TIME TERM}

In developing a technique for predicting the time required for long-term breakout, it was determined in the main text that a plot of the relative breakout force, $F_{b} / F_{I b}$, versus the breakout time normalized as $\left(p t_{b} / D^{2}\right)(B / D)^{2}$, yielded the best correlation. It was then observed that this normalized term could be nondimensionalized by multiplying it by $\mathrm{k} / \gamma$, where $k$ is the soil permeability in feet per minute and $\gamma$ is the unit weight of water in pounds per cubic feet. The objective of this appendix is to illustrate that a nondimensional term of this nature is not unreasonable from a soil mechanics point of view.

It will first be assumed that the mechanism of long-term breakout is the propagation of cracks down the side of the object. After the cracks have propagated to some point close to the object bottom, water will rush into the low-pressure zone beneath the object and breakout will occur. This assumption agrees well with what was observed.

The growth of the cracks appears associated with the flow of water either through the soil to the point at which the crack is growing or into the soil itself. If the water flows into the soil itself, the soil strength is reduced and the soil can flow more easily. Whatever the basic mechanism, one of the important relationships describing the phenomenon will be Darcy's law for flow through porous media. This empirical law can be written for plane flow as $^{14}$

$$
0=k h\left(\frac{N_{f}}{N_{d}}\right)
$$

$$
\text { where } \begin{array}{ll}
\mathrm{Q} & =\text { seepage rate per unit length }\left(\mathrm{ft}^{3} / \mathrm{min} / \mathrm{ft}\right) \\
\mathrm{k} & =\text { soil permeability }(\mathrm{ft} / \mathrm{min}) \\
\mathrm{h} & =\text { total pressure head loss }(\mathrm{ft})=\Delta \mathrm{p} / \gamma \\
\Delta \mathrm{p} & =\text { total pressure loss }(\mathrm{psf}) \\
\gamma & =\text { unit weight of water }(\mathrm{pcf}) \\
\mathrm{N}_{\mathrm{f}} / \mathrm{N}_{\mathrm{d}} & =\text { flow net factor, related to geometry of problem }
\end{array}
$$


For the breakout problem, the situation can be diagrammed as

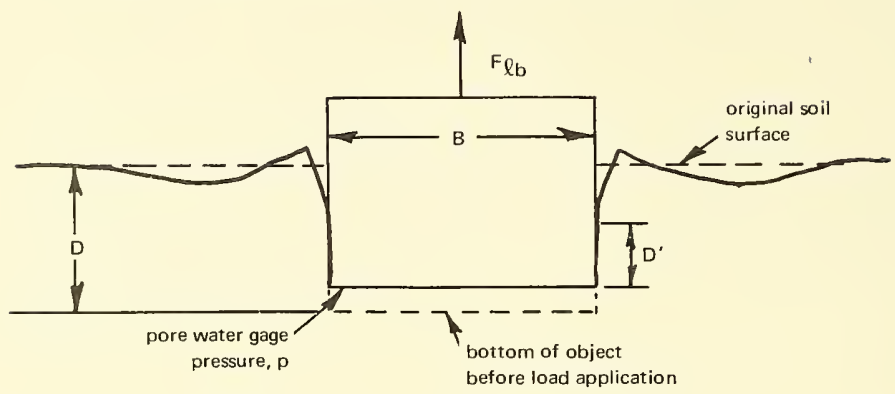

where $\mathrm{D}=$ original embedment depth

$D^{\prime}=$ portion of depth over which soil is in contact with object side after load application

$\mathrm{B}=$ object width

$F_{\ell_{b}}=$ line force on object

$\mathrm{p} \quad=$ gage pressure in pore water beneath object (equal to total pressure loss, $\Delta p$ ) $=F_{b} / A$

$F_{b}=$ force carried by soil $=F_{\ell b}-W_{b}+W_{s}$

$W_{\mathrm{b}}=$ weight of object in water

$W_{\mathrm{s}}$ = submerged weight of soil displaced by object

A $=$ cross-sectional area of object

Water flows under the influence of pressure, $p$, into the zone in which the soil is still in contact with the object side. The average flow rate, $\mathrm{Q}$, can be written as a volume of water per unit length, $v_{w}$, flowing over the time required for breakout, $t_{b}$.

$$
Q=\frac{V_{w}}{t_{b}}
$$

The volume of water per unit length which must flow before breakout will occur is clearly proportional to the portion of embedment depth which is in contact with the object side, $\mathrm{D}^{\prime}$. This volume is also proportional to some 
characteristic horizontal length which represents either the ultimate average crack width or the width of the zone of soil influenced by water seeping into it. This characteristic length may be proportional to $D^{\prime}$. If so, that would indicate that the deeper the object is embedded the greater will be the required crack width or the greater the zone of influence with regard to the surrounding soil. If this is assumed,

$$
Q \propto \frac{D^{\prime 2}}{t_{b}}
$$

Equation $\mathrm{C}-1$ can be rewritten as the relationship

$$
\frac{D^{\prime 2}}{t_{b}} \propto k \frac{p}{\gamma}\left(\frac{N_{f}}{N_{d}}\right)
$$

The portion of the original embedment depth still in contact with the object side, D', will vary inversely with the upward displacement which occurs when the force is applied and directly with the original embedment depth, D. The upward displacement was observed to vary directly with the relative force applied, $\mathbf{F}_{\mathbf{b}} / \boldsymbol{F}_{\mathrm{Ib}}$. Therefore,

$$
D^{\prime} \propto D f\left(\frac{F_{I b}}{F_{b}}\right)
$$

where $f()=$ a functional relationship

Flow net factors, $\hat{N}_{f} / N_{d}$, are characteristically related to nondimensionalized geometry terms. One such term for this problem would be B/D.

$$
\frac{N_{f}}{N_{d}}=g\left(\frac{B}{D}\right)
$$

where $g()=$ a functional relationship

Relationship C-2 can be rewritten and its terms reordered as

$$
j\left(\frac{F_{b}}{F_{I b}}\right) \propto t_{b} \frac{k p}{\gamma D^{2}} g\left(\frac{B}{D}\right)
$$


where $j\left(\frac{F_{b}}{F_{I b}}\right)=$ function of $\frac{F_{b}}{F_{I b}}=\frac{1}{\left[f\left(\frac{F_{I b}}{F_{b}}\right)\right]^{2}}$

Relationship C-3 has the same form as Equation 7 of the main text and thereby lends additional reliability to it.

\section{REFERENCES}

1. Naval Civil Engineering Laboratory. Technical Note N-1133: Specialized anchors for the deep sea; progress summary, by J. E. Smith, R. M. Beard, and R. J. Taylor. Port Hueneme, Calif., Aug. 1970. (AD 716408)

2.- Contract Report CR-69.031: Breakout resistance of objects embedded in ocean bottom, by A. S. Vesic. Durham, N. C., May 1969. (Contract no. N62399-68-C-0043) (AD 699525)

3. - Evaluation of bottom breakout reduction methods, by K. D. Vaudrey. Port Hueneme, Calif. (To be published)

4. Lockheed Missiles and Space Company. Large object recovery technology development/breakout force reduction program, by R. D. Grunert. Sunnyvale, Calif. (To be published)

5. Naval Civil Engineering Laboratory. Technical Note N-863: Breakout forces, by B. J. Muga. Port Hueneme, Calif., Sept. 1966. OUO

6. - Technical Report R-591: Ocean bottom breakout forces, including field test data and the development of an analytical method, by B. J. Muga. Port Hueneme, Calif., June 1968. (AD 837647L)

7.- Technical Report R-635: Ocean sediment holding strength against breakout of embedded objects, by C. L. Liu. Port Hueneme, Calif., Aug. 1969. (AD 692411)

8. Southwest Research Institute. Report on Contract N-Onr-336300: Force required to extract objects from deep ocean bottom, by R. C. DeHart and C. R. Ursell. San Antonio, Tex., Sept. 1967. (AD 658757)

9. Lockheed Missiles and Space Company. Report No. LMSC 73108:

Breakout force test program. Santa Cruz, Calif., Dec. 1967.

10. A. W. Skempton. "The bearing capacity of clays," in Building Research Congress, London, Collected papers, div. 1, pt. 3, Sept. 1951, pp. 180-189. 
11. D. W. Taylor. Fundamentals of soil mechanics. New York, Wiley, 1948, p. 573.

12. T. W. Lambe and R. V. Whitman. Soil mechanics. New York, Wiley, 1969, p. 433.

13. K. Hoeg, J. T. Christian, and R. V. Whitman. "Settlement of strip load on elastic-plastic soil," American Society of Civil Engineers, Proceedings, Journal of the Soil Mechanics and Foundations Division, vol. 94, no. SM2, Mar. 1968, pp. 431-445.

14. J. L. Sherard, et al. Earth and earth-rock dams; engineering problems of design and construction. New York, Wiley, 1963, p. 275. 
NOMENCLATURE

A

B

D

$D^{\prime}$

$F_{b}$

$F_{\text {Ib }}$

$F_{\ell b}$

$\mathrm{F}_{\text {lIb }}$

$F_{l w}$

$F_{q}$

$F_{q}^{\prime}$

h

k

$\mathrm{L}$
Cross-sectional area of object at soil-water interface

Width of object at soil-water interface

Embedment depth of object before force application $\left(\mathrm{V}_{\mathrm{s}} / \mathrm{A}\right)$

Portion of embedment depth along which soil-object contact is maintained after force application

Portion of applied line force carried by soil $\left(F_{l b}-W_{b}+W_{s}\right)$

Portion of immediate breakout line force carried by soil $\left(F_{\ell I b}-W_{b}+W_{s}\right)$

Upward line force applied to object to cause breakout

Upward line force applied to object to cause immediate breakout

Upward line force applied to object during waiting period

Downward force carried by soil during waiting period $\left(W_{b}+W_{s}-F_{\ell w}\right)$ (for cases in which bearing capacity is exceeded during object placement)

Bearing capacity force soil is capable of maintaining $[6 \mathrm{AS}(1.0+0.2 \mathrm{D} / \mathrm{B})(1.0+0.2 \mathrm{~B} / \mathrm{L})]$

Hydraulic head difference between top and bottom of object $(\mathrm{p} / \gamma)$ (length)

Permeability of soil (length/time)

Length of object 


\begin{tabular}{|c|c|}
\hline$N_{f} / N_{d}$ & Dimensionless flow net factor \\
\hline $\mathbf{P}$ & Bearing capacity force \\
\hline $\mathrm{p}$ & Gage pressure in pore water beneath object $\left(F_{b} / A\right)$ \\
\hline $\mathbf{Q}$ & $\begin{array}{l}\text { Rate of water flow into zone below object (per } \\
\text { unit length) (length } 3 / \text { time/length) }\end{array}$ \\
\hline$r, \theta, r_{0}$ & $\begin{array}{l}\text { Parameters of log spiral in solution to Prandtl's } \\
\text { equation }\end{array}$ \\
\hline S & Undrained shear strength of soil (force/length ${ }^{2}$ ) \\
\hline $\mathrm{T}$ & Normalized breakout time $\left[\frac{p t_{b}}{D^{2}}\left(\frac{B}{D}\right)^{2}\right]\left(\frac{\text { time-force }}{\text { length }^{4}}\right.$ \\
\hline$t_{b}$ & Time required for breakout \\
\hline$t_{w}$ & $\begin{array}{l}\text { Waiting time after object placement and before } \\
\text { application of } F_{\ell_{b}} \text { or } F_{\ell \mathbf{b}}\end{array}$ \\
\hline$v_{s}$ & Volume of soil displaced by object \\
\hline$v_{w}$ & Volume of water per unit length \\
\hline$W_{b}$ & Weight of object in water \\
\hline$W_{s}$ & Buoyant weight of soil displaced by object \\
\hline$f(), g(), j()$ & Functional relationships \\
\hline$\Delta \mathrm{p}$ & Total pressure loss (force/length ${ }^{2}$ ) \\
\hline$\gamma$ & Unit weight of seawater \\
\hline$\gamma_{\mathrm{s}}$ & Buoyant unit weight of soil \\
\hline$\phi$ & Friction angle of soil \\
\hline
\end{tabular}





\section{DISTRIBUTION LIST}

$\begin{array}{cccc}\begin{array}{c}\text { SNDL } \\ \text { Code }\end{array} & \begin{array}{c}\text { No. of } \\ \text { Activities }\end{array} & \begin{array}{c}\text { Total } \\ \text { Copies }\end{array} & \\ - & 1 & 12 & \text { Defense Documentation Center } \\ \text { FKAIC } & 1 & 10 & \text { Naval Facilities Engineering Command } \\ \text { FKNI } & 6 & 6 & \text { NAVFAC Engineering Field Divisions } \\ \text { FKN5 } & 9 & 9 & \text { Public Works Centers } \\ \text { FA25 } & 1 & 1 & \begin{array}{l}\text { Public Works Center } \\ \text { RDT\&E Liaison Officers at NAVFAC } \\ -\end{array} \\ & 9 & 9 & \begin{array}{l}\text { Engineering Field Divisions and } \\ \text { Construction Battalion Centers } \\ \text { NCEL Special Distribution List No. 9 } \\ \text { for persons and activities interested in } \\ \text { reports on Deep Ocean Studies }\end{array}\end{array}$





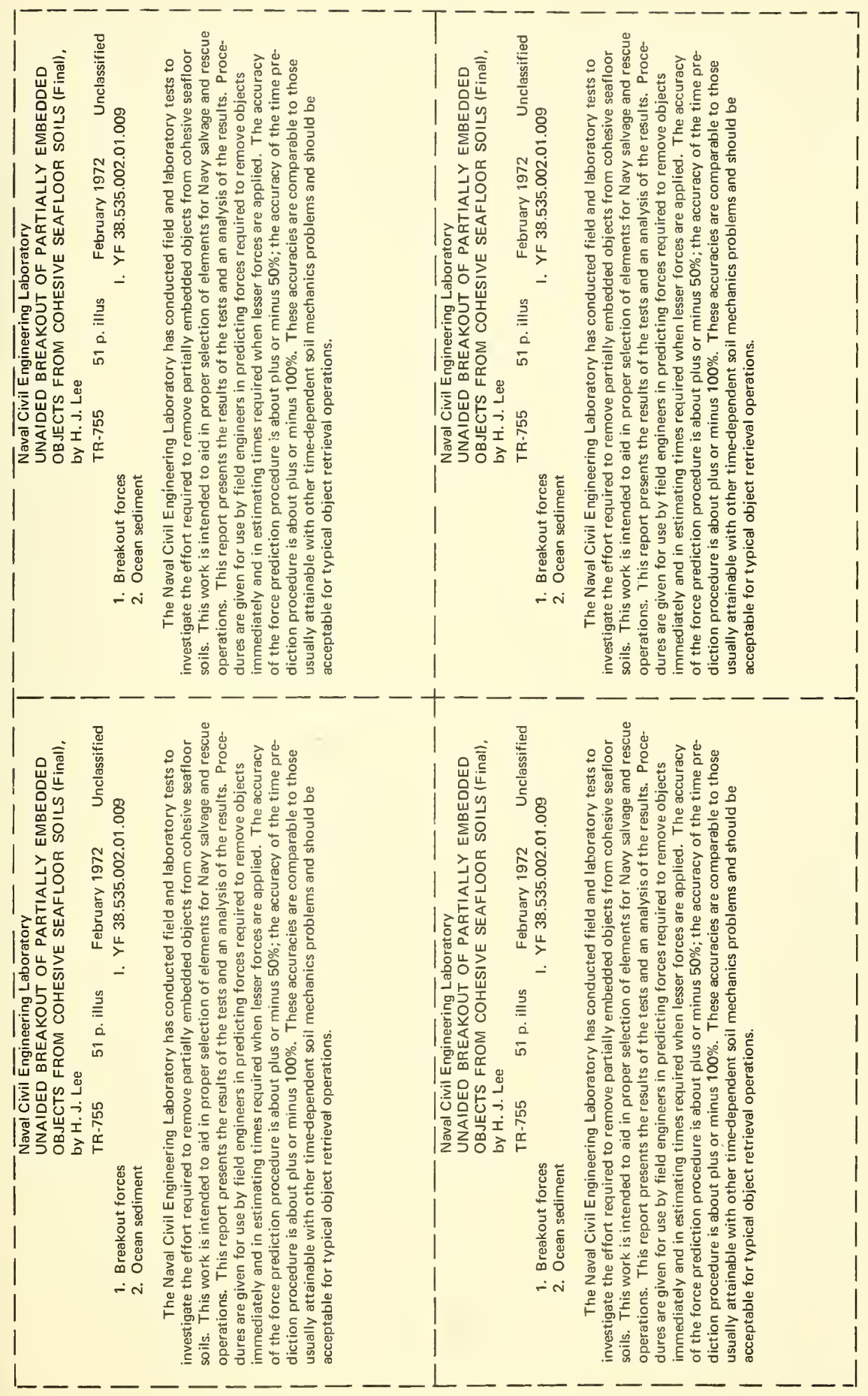





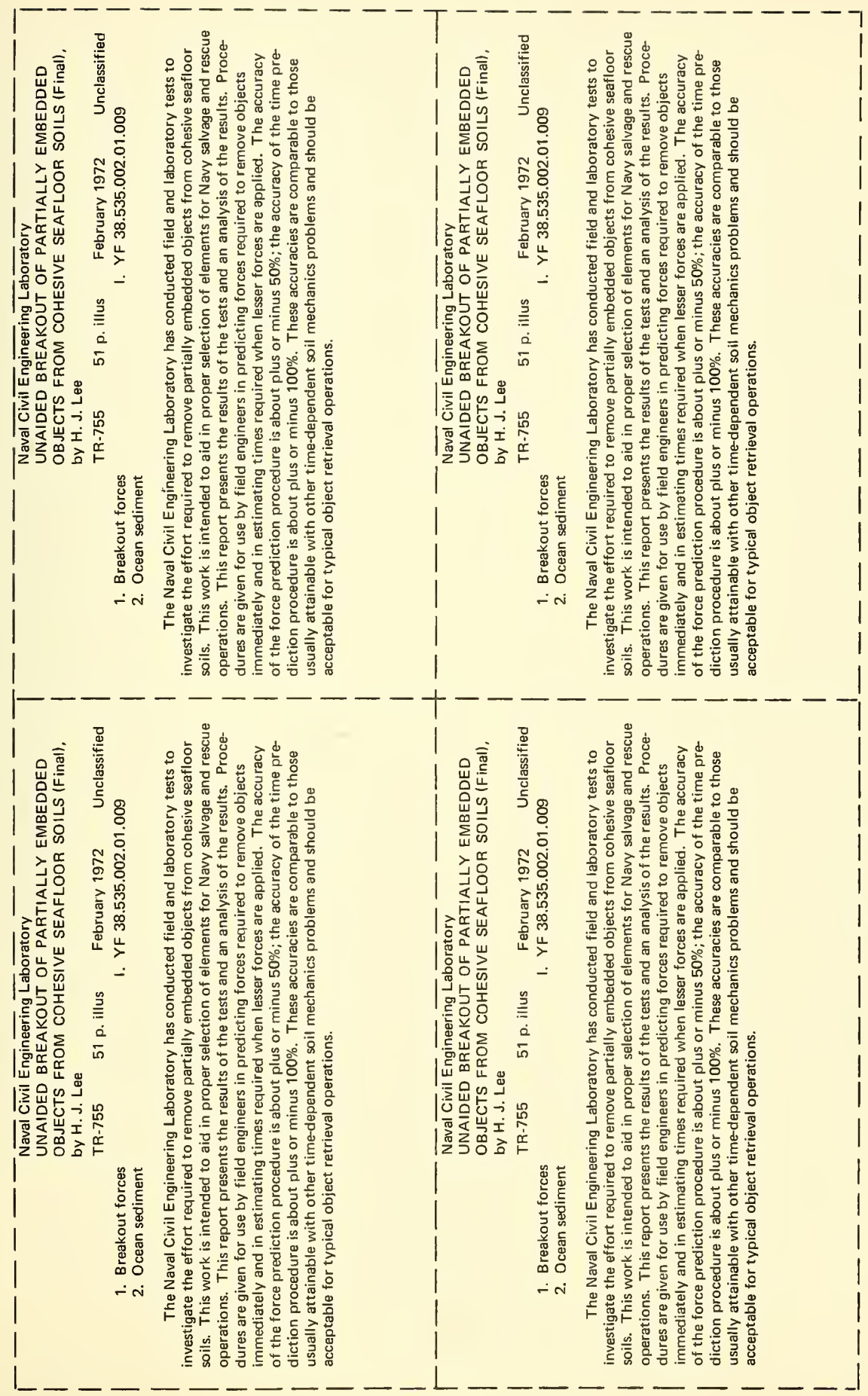


Unclassified

Secunty Classification

DOCUMENT CONTROL DATA - R \& D

(Securtly classilication of titie, body of abstrart and indexang annotation nust be ontered when the overell report is classilied)

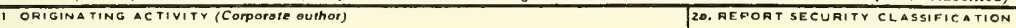

Naval Civil Engineering Laboratory

Port Hueneme, California 93043

Unclassified

\section{REPOAT TITLE \\ UNAIDED BREAKOUT OF PARTIALLY EMBEDDED OBJECTS FROM COHESIVE SEAFLOOR SOILS}

4. OESCRIPTIVE NOTES (TYPA of report and inclusive datea)

Final; $1965-1971$

S AU THOR(S) (Firat name, middlo inllial, iAat namo)

H. J. Lee

\begin{tabular}{|c|c|c|}
\hline $\begin{array}{l}\text { 6. AEFORT OATE } \\
\text { February } 1972\end{array}$ & $\begin{array}{l}\text { 7a. TOTAL NO OF DAGES } \\
51\end{array}$ & $\begin{array}{r}\text { 7b. NO. OF AEFS } \\
14\end{array}$ \\
\hline $\begin{array}{l}\text { BA. CONTRACTOA GRANT NO. } \\
\text { B. PROJECT NO. YF } 38.535 .002 .01 .009\end{array}$ & \multicolumn{2}{|c|}{$\begin{array}{l}\text { 9a. ORIGINATOR'S REPORT NUMEER(S) } \\
\text { TR-755 }\end{array}$} \\
\hline $\begin{array}{l}\text { c. } \\
\text { d. }\end{array}$ & \multicolumn{2}{|c|}{ 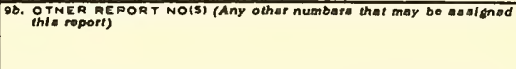 } \\
\hline
\end{tabular}

Approved for public release; distribution unlimited.

\begin{tabular}{|l|l|}
\hline 11. SUPALEMENTARY NOTES & $\begin{array}{l}\text { 12. SPONSORING MILITAAY ACTIVITY } \\
\text { Naval Facilities Engineering Command } \\
\text { Washington, D. C. } 20390\end{array}$ \\
\hline I3 ABSTAACT & \begin{tabular}{l} 
Wat \\
\hline
\end{tabular}
\end{tabular}

The Naval Civil Engineering Laboratory has conducted field and laboratory tests to investigate the effort required to remove partially embedded objects from cohesive seafloor soils. This work is intended to aid in proper selection of elements for Navy salvage and rescue operations. This report presents the results of the tests and an analysis of the results. Procedures are given for use by field engineers in predicting forces required to remove objects immediately and in estimating times required when lesser forces are applied. The accuracy of the force prediction procedure is about plus or minus $50 \%$; the accuracy of the time prediction procedure is about plus or minus $100 \%$. These accuracies are comparable to those usually attainable with other time-dependent soil mechanics problems and should be acceptable for typical object retrieval operations.

\section{DD, FORM 1473 (PAGE 1)}

$S / N 0101.807 .6801$
Unclassified

Security Classification 
Unclassified

Secursty Classification

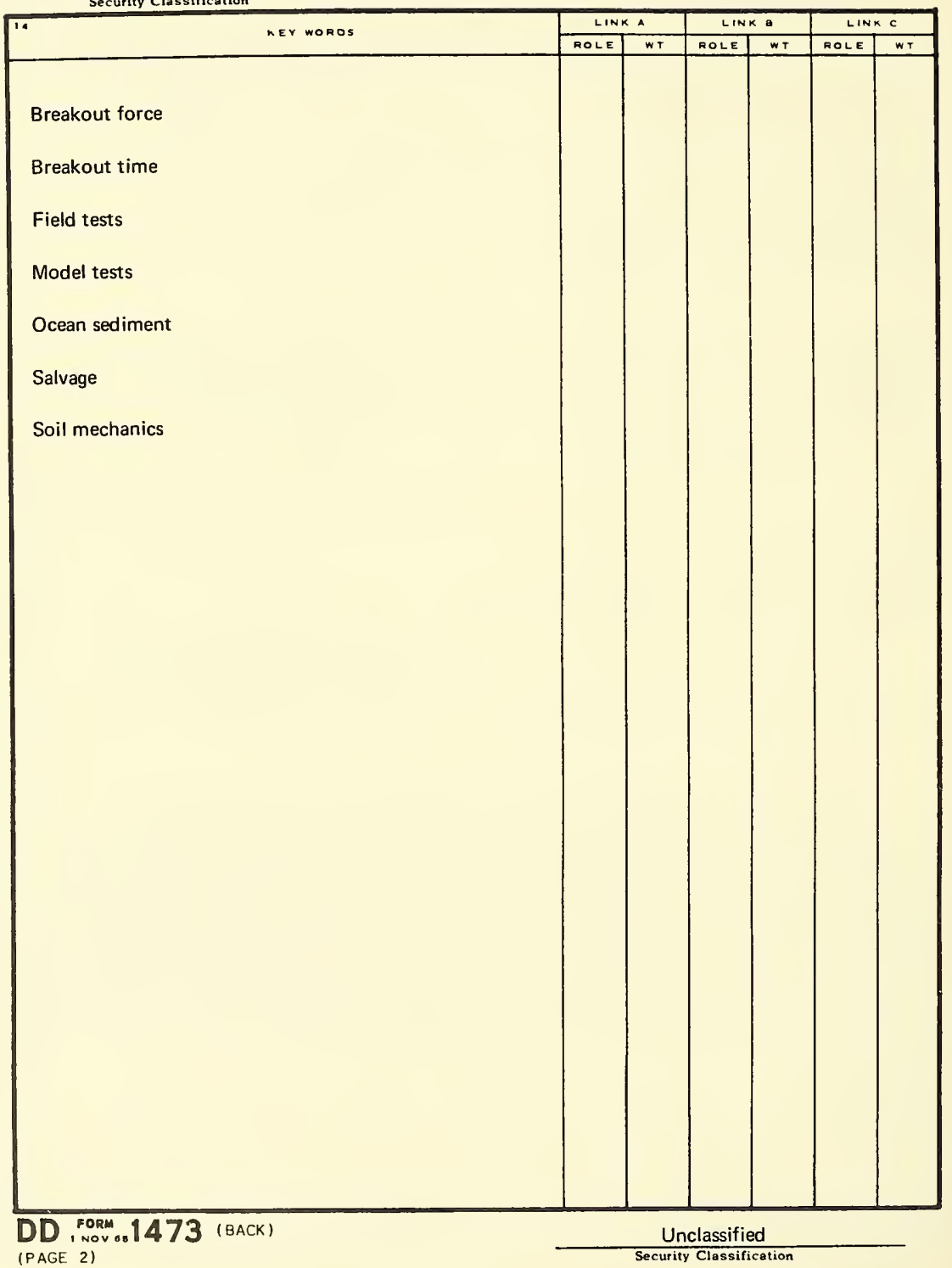



